\title{
Eigenoscillations of the differentially rotating Sun
}

\section{22-year, 4000-year, and quasi-biennial modes}

\author{
N. S. Dzhalilov ${ }^{1,2}$, J. Staude ${ }^{2}$, and V. N. Oraevsky ${ }^{1}$ \\ 1 Institute of Terrestrial Magnetism, Ionosphere and Radio Wave Propagation of the Russian Academy of Sciences, \\ Troitsk City, Moscow Region 142190, Russia \\ e-mail: namig@izmiran.rssi.ru; oraevsky@izmiran.rssi.ru \\ 2 Astrophysikalisches Institut Potsdam, Sonnenobservatorium Einsteinturm, 14473 Potsdam, Germany
}

Received 27 June 2001 / Accepted 20 December 2001

\begin{abstract}
Retrograde waves with frequencies much lower than the rotation frequency become trapped in the solar radiative interior. The eigenfunctions of the compressible, nonadiabatic ( $\epsilon$-mechanism and radiative losses taken into account) Rossby-like modes are obtained by an asymptotic method assuming a very small latitudinal gradient of the rotation rate. An integral dispersion relation for the complex eigenfrequencies is derived as a solution of the boundary value problem. The discovered resonant cavity modes (called $R$-modes) are fundamentally different from the known $r$-modes: their frequencies are functions of the solar interior structure, and the reason for their existence is not related to geometrical effects. The most unstable $R$-modes are those with periods of $\approx 1-3 \mathrm{yr}, 18-30 \mathrm{yr}$, and 1500-20 $000 \mathrm{yr}$; these three separate period ranges are known from solar and geophysical data. The growing times of those modes which are unstable with respect to the $\epsilon$-mechanism are $\approx 10^{2}, 10^{3}$, and $10^{5}$ years, respectively. The amplitudes of the $R$-modes are growing towards the center of the Sun. We discuss some prospects to develop the theory of $R$-modes as a driver of the dynamics in the convective zone which could explain, e.g., observed short-term fluctuations of rotation, a control of the solar magnetic cycle, and abrupt changes of terrestrial climate in the past.
\end{abstract}

Key words. hydrodynamics - Sun: activity - Sun: interior - Sun: oscillations - Sun: rotation

\section{Introduction}

The 22-year magnetic cycle of solar activity is the most prominent phenomenon of several large-scale dynamic events that occur in the Sun. (Really, the magnetic half cycles or sunspot number cycles vary in length between $9-13$ years, and $11 \mathrm{yr}$ is an average of the $\approx 20$ half-cycles available.) An explanation of the basic mechanism underlying this fameous phenomenon is the fundamental challenge of solar physics. The achievements of the theory of the $\alpha-\omega$ dynamo turned out to be a great success. However, neither all observations of magnetic and flow fields nor the radiation fluxes which are related to this phenomenon and which are measured at the surface of the Sun or indirectly, by helioseismology, in its interior, can be explained unambigously in this way. Although our present work is not directly related to the dynamo theory, we will outline here those difficulties which have common points with our results.

Send offprint requests to: J. Staude, e-mail: jstaude@aip.de

\subsection{Some problems of dynamo theory}

As a consequence of our imperfect knowledge of basic characteristics of turbulent convection as well as meridional circulation and details of the rotation of the Sun's interior, the solutions of the dynamo equations become functions of many free, unknown parameters (e.g. Stix 1976). For instance, by clever combinations of these parameters it is possible to get from kinematic theory an oscillatory magnetic field with a 22-year period and a growing amplitude. However, another choice of these parameters leads to waves of growing amplitude for other periods. So one could draw a butterfly diagram not only with an 11-year periodicity. It remains still an open question which of the clever combinations resulting in a solar-like 22-year activity cycle is realized in the Sun. We could not find a work on the dynamo wave problem, showing that just the 22 -year period is preferred among others with a maximum growth rate and with the spatial scales required for solar activity. Instead, many authors pointed out that the cycle period of 22 years is hard to explain (Stix 1991; 
Gilman 1992; Levy 1992; Schmitt 1993; Brandenburg 1994; Weiss 1994; Rüdiger \& Arlt 2000).

From the solution of the inverse problem of helioseismology (e.g. Tomczyk et al. 1995) it is known that the convective envelope of the Sun is rotating with a latitude dependence of the angular velocity similar to that of the surface but almost rigid in radial direction. A stronger radial gradient which is required for the $\alpha-\omega$ dynamo mechanism is located in a shallow layer (thickness $\approx 0.05 R_{\odot}$ (Kosovichev 1996), where $R_{\odot}$ is the solar radius) immediately below the convective zone - the tachocline (Spiegel \& Zahn 1992). Below the tachocline up to a depth of at least $0.5 R_{\odot}$ the radiative interior is rotating with an angular velocity law similar to that of a solid-body. The question arises: what compels the Sun to rotate in such a strange manner, which is different from the generally accepted, theoretically predicted stable rotation law? How to handle a dynamo theory for which the " $\omega$ " area is separated from the " $\alpha$ " area over a large part of the extent of the convective zone? In order to solve this problem Parker (1993) has put forward the idea of an interface dynamo, the basic features of which existed already in earlier dynamo models (Steenbeck et al. 1966). To close the cycle of such a stretched dynamo it is necessary to have some mechanism delivering toroidal magnetic flux, arising by the shear of differential axisymmetric rotation (Cowling 1953) in the tachocline, to the " $\alpha$ " dynamo area (e.g. Moffatt 1978; Krause \& Rädler 1980). To get a solar-like magnetic activity it is necessary to suppose the existence of a huge $\left(\approx 10^{5} \mathrm{G}\right)$ toroidal magnetic field to create enough magnetic buouancy for the leakage of magnetic flux and to solve the tilt problem of lifting loops (e.g. Caligari et al. 1998). Moreover, a high magnetic diffusivity contrast between the convective envelope and the underlying radiative core should be assumed to solve the quenching problem of the $\alpha$ effect (see, e.g., Fan et al. 1993; Cattaneo \& Hughes 1996). However, it is a major challenge for any dynamo model to produce such strong fields.

The idea of the interface dynamo was further developed, e.g. by Charbonneau \& MacGregor (1997). Later, a fit to the real solar rotation profile with its latitudinal and radial dependencies has been included by Markiel \& Thomas (1999), but so far no satisfactory solar-like oscillatory solutions for the interface dynamo have been found. Growing wave solutions are suppressed by the latitudinal shear.

\subsection{Spinning-down rotation problem}

Mechanisms for braking the solar internal rotation are also under discussion. The character of the core rotation is not clear because here the accuracy of helioseismic inversions gets worse (Chaplin et al. 1999) and the results seem to be in contradiction with the oblateness measurements (Paterno et al. 1996). There are some suggestions that a deceleration of the radiative interior depends on the transport of angular momentum between this region and the convective zone. For instance, Mestel \& Weiss (1987) supposed that even a weak large-scale magnetic field would be sufficient to couple very efficiently the interior and the convective zone, leading essentially to solid body rotation. In this way the magnetic torques can also extract angular momentum from the radiative interior (e.g. Charbonneau \& MacGregor 1993).

The wave mechanism for the solution of this problem is more popular. Schatzman (1993), Zahn et al. (1997), and Kumar \& Quataert (1997) have concluded that the solid rotation of the radiative interior is a direct consequence of the effect of internal gravity waves. Gravity waves generated near the interface between the convective and radiative regions transport retrograde angular momentum into the interior, thereby spinning it down. Here the main idea is that the isotropically generated gravity waves become anisotropic due to Doppler shifts of frequencies in the differentially rotating Sun. In that way for anisotropic retrograde and prograde waves the radiative damping is different, and the residual negative angular wave momentum may compel the solar radiative interior to co-rotate with the convective zone. This idea has been further developed by Kumar et al. (1999) including a toroidal magnetic field to explain the existence of the unstable shear layer "tachocline". However, Ringot (1998) has shown that a quasi-solid rotation of the radiative interior cannot be a direct consequence of the action of internal gravity waves produced in the convective zone. Gough (1997) questioned this idea emphasizing that the mechanism can work only if the waves are generated with strong amplitudes to transport the required angular momentum. This means, resonance waves are required, but such waves may penetrate only to distances less than $10^{-5} R_{\odot}$ beneath the convective zone due to the strong radiative damping. These waves must deposit their angular momentum before returning to the convective zone, but not before penetrating far into the radiative interior.

For the wave mechanism the question of an anisotropic propagation relative to the azimuthal rotation is a key moment. Fritts et al. (1998) have shown that convection, penetrating into the stratified and strongly sheared tachocline, can produce preferentially propagating gravity waves.

There have also been speculations that the rotation of the core may be variable, perhaps with a time scale of the solar cycle (e.g. Gough 1985). The present paper is along these lines.

From our short discussion we conclude that the convective envelope and the radiative interior are coupled to each other through a certain global agent, resulting in almost co-rotation. To advance the solution of the problem the dynamo theory should take into account the presence of this global agent. We suppose that really this agent is provided by waves with the following properties:

Waves should represent large-scale global eigenoscillations of the Sun. Their origin must be related to rotation, they must be strongly anisotropic with respect to the azimuthal angle. Looking at the characteristics of the 
solar cycle we immediately see the high coherency of these global motions (the constant periods, phase shifts, amplitudes, the latitude appearence, etc.). Activity grows in the first phase with a timescale which is considerably shorter than the decay time in the second phase; this fact and the quick eruptive release of energy by the reconnection mechanism indicate that the waves must be unstable.

It is noteworthy that the inner gravity waves do not fulfill these requirements. The quesion is whether the $r$ modes do?

\section{3. $r$-modes}

In a non-rotating sphere $(\Omega=0$, where $\Omega$ is the angular frequency of solar rotation) the wave motion is subdivided into two non-coupling components: spheroidal $p-$, $f-$ and $g$-modes (for which the main restoring forces are pressure gradient and buouancy) and toroidal modes (e.g. Unno et al. 1989). Toroidal modes are degenerated horizontal eddy motions confined to a spherical surface with a radius $r$ for which $\omega=0, \operatorname{div} \boldsymbol{v}=0$, and $\boldsymbol{v}=Q_{l}^{m}(r) \times\left(0, \frac{1}{\sin \theta} \frac{\partial}{\partial \phi},-\frac{\partial}{\partial \theta}\right) Y_{l}^{m}(\theta, \phi)$. Here $Y_{l}^{m}$ is the spherical harmonic with a degree $l$ and order $m, \theta$ is the colatitude, $\phi$ is the azimuthal angle in the spherical polar coordinates, $\boldsymbol{v}$ is the fluid velocity field, $\omega$ is the angular frequency of the fluid motion, and $Q_{l}^{m}(r)$ is an arbitrary amplitude function. Toroidal modes have zero radial velocity but have non-zero radial vorticity, $(\operatorname{rot} v)_{\mathrm{r}} \neq 0$ (for the spheroidal modes it is vice versa). These modes do not alter the equilibrium configuration.

When a slow rotation $\left(\Omega^{2}<\Omega_{\mathrm{g}}^{2}=G M / R^{3}\right)$ is included the spheroidal modes are slightly modified but they keep their main properties. Degeneracy of toroidal modes is removed only partially by the rotation, and quasi-toroidal waves - known as $r$-modes - appear with a non-zero frequency of $\omega \approx 2 \Omega \mathrm{m} / l(l+1)$ in the rotating frame (Papaloizou \& Pringle 1978; Brayn 1889). Usually the governing equations of the $r$-modes are obtained by expanding the initial physical variables of the equations in the rotating system into power series with respect to the small parameter $\left(\Omega / \Omega_{\mathrm{g}}\right)^{2}\left(\approx 10^{-4.7}\right.$ for the Sun, e.g. Papaloizou \& Pringle 1978; Provost et al. 1981; Smeyers et al. 1981; Saio 1982). These power series practically describe the deviation of the surface of the star from its initial spherical state, resulting from rotation through Coriolis and centrifugal forces. As a result of the deformation of the spherical surface with a radius $r$ the radial vorticity of the toroidal modes cause a surface pressure perturbation through the Coriolis force. However, the $r$-modes practically keep the main properties of toroidal flows: $v_{\mathrm{r}} \approx 0, \operatorname{div} \boldsymbol{v} \approx 0$. The degeneracy of the $r$-modes is that their frequencies hardly depend on $Q_{l}^{m}(r)$, i.e. they are independent from the inner structure of the star. For the $l=1$ modes the frequency in the inertial system is again close to zero, $\omega \approx 0$ (Papaloizou \& Pringle 1978). The $r$-mode equations define the amplitudes $Q_{l}^{m}(r)$, and taking into account the next terms with small $\Omega^{2} / \Omega_{\mathrm{g}}^{2}$ in the series practically does not change the frequencies.

Due to the fact that the $r$-modes are practically surface deformation waves, some similarity of these waves to the surface gravity waves or to the $f$-modes is apparent. For high $l$ the $f$-modes are an analogy to surface gravity waves in a plane-parallel fluid with $\omega^{2}=g k_{\perp}$. In the Cowling approximation $f$-modes with $l=1$ have zero frequency too, $\omega \approx 0$ (Unno et al. 1989). This corresponds to a parallel displacement of the whole star. For high $l$ the $f$-mode frequencies are also independent of the inner structure, with $\omega^{2} \approx l \Omega_{\mathrm{g}}^{2}$ (Gough 1980). So, $r$-modes are also fundamental rotating modes with an inertial frequency, $\omega \leq 2 \Omega$.

For the Sun the properties of $r$-modes have been investigated in great detail by Wolff et al. (1986) and Wolff (1998, 2000; and Refs. therein).

Some properties of the $r$-modes are also similar to those of the Rossby waves in geophysics (Pedlosky 1982). Similar to the Rossby waves and unlike the $g$-modes the $r$-modes are strongly anisotropic. They propagate only in azimuthal direction, opposite to rotation (i.e. they are retrograde waves in the co-rotating frame). Because we are interested in length scales corresponding to those of large sunspots, we have to consider $r$-modes with $l \approx 100$. To get oscillations with periods of years $\left(\omega / \Omega \approx 10^{-2}\right)$ we must choose $m \approx l \gg 1$, just such $r$-modes are physically more interesting (Lockitch \& Friedman 1999). However, in the case of high $l$ the amplitudes of the $r$-modes will be concentrated near the surface of the Sun (Provost et al. 1981; Wolff 2000), and so they can actively interact with convective motions (Wolff 1997, 2000). Because for these modes $v_{\mathrm{r}} \approx 0$ and $\operatorname{div} \boldsymbol{v} \approx 0$, their chance to take part in the redistribution of angular rotation momentum in the radiative interior is low. Note that the slow solar differential rotation does not change the behavior of such $r$-modes with $m=l \gg 1$ (Wolff 1998).

Looking for further analogies between waves connected with gravity and with rotation, we remember that beside the surface gravity waves there exist internal gravity waves with $\omega^{2} \approx N^{2} k_{x}^{2} /\left(k_{x}^{2}+k_{y}^{2}\right)$, the frequencies of which depend on the inner structure $(N$ is the Brunt-Väisälä frequency). Similar to these waves there exist "true" Rossby (not deformation) waves, the frequency of which depends also on the internal structure.

\subsection{Rossby waves}

We include here a short review on the main features of Rossby waves; they have been investigated in great detail in geophysics (e.g. Pedlosky 1982; Gill 1982). In the simplest case, that is in a plane-parallel, homogeneous, rotating layer, the dispersion relation for the Rossby waves is $\omega \approx 2 \Omega \beta k_{x} /\left(k_{x}^{2}+k_{y}^{2}+k_{z}^{2}\right)$. Here $k_{x}$ is the wave number perpendicular to the rotation axis, $k_{z}$ is expressed by the internal deformation radius of Rossby which depends on the Brunt-Väisälä frequency, $\beta$ is the transverse gradient (in $y$ direction) of the Coriolis parameter: a vertical 
component of the "planetary" vorticity $2 \boldsymbol{\Omega}$ in the given local point. Unlike the $r$-modes the Rossby wave frequencies are functions of the internal structure and have maximum dependence on the gradient $\beta: \omega \rightarrow 0$ if $k_{x} \rightarrow 0$ and if $k_{x} \rightarrow \infty$.

Any disturbance of the local flow in a rotating frame may generate waves of the Rossby type. These waves exist only if there is a gradient of the potential vorticity $\boldsymbol{\Pi}=$ $\left(\boldsymbol{\omega}_{\mathrm{a}} \cdot \nabla \Xi\right) / \rho$. Here an absolute vorticity is the sum of the relative and the planetary vorticities, $\boldsymbol{\omega}_{\mathrm{a}}=\operatorname{rot} \boldsymbol{v}+2 \boldsymbol{\Omega}, \boldsymbol{\Xi}$ is any conserved scalar quantity, $\frac{\mathrm{d} \Xi}{\mathrm{d} t}=0$ (for instance, for adiabatic motion that could be the entropy or the density in the case of incompressible plasma). The Rossby wave motion is a solution of the nonlinear equation for transport of $\Pi$. The potential vorticity is conserved if the medium is barotropic $(\nabla \rho \times \nabla p=0)$ and if there are no torques. The rotation of the frame is added to any vorticity in the velocity field. Any motion within a rotating fluid serves as a potential source for vorticity.

The relative vorticity may be evoked by the geometrical surface as well as by internal gradients. It depends on the choice of the function $\Xi(r, \theta, \phi)$ and on $\boldsymbol{\Omega}(r, \theta)$. For example, an unevenness of the ocean bottom causes the topographic Rossby waves, or a dependence of the Coriolis parameter on the earth latitude $(F=2 \Omega \sin \varphi$, where $\varphi$ is the geographic latitude) is the main cause of atmospheric Rossby waves.

In the solar dynamo context the ability of Rossby waves to induce solar-like magnetic fields has been considered by Gilman (1969). Here the mechanism for sustaining the Rossby waves is a latitudinal temperature gradient in a thin, rotating, incompressible convective zone. To interpret the dynamical features of large-scale magnetic fields the Rossby vorticies excited within a thin layer beneath the convective zone are considered by Tikhomolov \& Mordvinov (1996) as the result of a deformation of the lower boundary of the convective zone.

\subsection{R-modes}

From the discussion in Sect. 1.4 we conclude that just Rossby-like waves could be suitable for our requirements. As the main driving mechanism we choose a latitudinal (or horizontal) differential rotation, $\Omega=\Omega(\theta)$. Baker \& Kippenhahn (1959) have pointed out that the uniform rotation of a star is not a typical case. Low frequencies (periods of years) could easily be obtained searching for the eigenoscillations of the Sun's radiative interior, where the gradient of the rotation speed is close to zero (in accordance with the helioseismology results). Large scales such as those associated with sunspots $\left(k_{x} R_{\odot} \sim 100\right)$ decrease the frequencies too. Similar to the $r$-modes the Rossby waves are strongly anisotropic (retrograde waves), but unlike the $r$-modes these waves are concentrated close to the solar center. These results have already been obtained by Oraevsky \& Dzhalilov (1997), who investigated the trapping of adiabatic, incompressible Rossby-like waves in the solar interior. In the present work we take into account compressibility for the nonadiabatic waves. We look for unstable waves. It is clear that the necessary condition for the Kelvin-Helmholtz shear instability, $4 N^{2}-\left(r \frac{\mathrm{d} \Omega}{\mathrm{d} r}\right)^{2}<0$ (Ando 1985), is not fulfilled in the radiative interior. Then we decided to include the thermal $\varepsilon$-mechanism of instability which is favoured at low frequencies (Unno et al. 1989). To balance the $\varepsilon$-mechanism the radiative losses in the diffusion regime are included. To exclude all geometrical effects we ignore the influence of the spherical surface at the given radius. The dispersion relation in the limit of adiabatic incompressiblity and at very low frequencies is the same as that for Rossby waves in geophysics. In order to distinguish these rotational body waves from the $r$-modes we call them $R$-modes (Rossby rotation).

The governing fourth order equation is obtained from the basic equations in Sect. 2. Some qualitative analysis of the wave cavity trapping is done for the simpler adiabatic case in Sect. 3. Using the asymptotic solutions obtained in Sect. 4 the complex boundary value problem is solved in Sect. 5. The calculation of the eigenfrequencies and the instability analysis are done in Sect. 6 . The obtained unstable modes are shortly discussed in Sect. 7.

\section{Setting the problem}

\subsection{Basic equations}

Let us investigate global motions with large timescales such that the Rossby number is small, $\omega / \Omega<1$. Before the appearence of any disturbances the basic stationary state of the rotating star is defined mainly by the balance of pressure gradient, gravity force, and forces exerted by the noninertiality of the motion (Coriolis and centrifugal forces). In the case of an incompressible fluid with a homogeneous rotation rate usually this state is called "geostrophic balance". A star disturbed by an external force tends to return to this basic state. Our aim is to study for the Sun the dynamics of small deviations from the steady geostrophic balance. For this purpose it is natural to write the dynamic equations in a frame rotating together with the Sun. The magnetic field will be ignored. For arbitrary $\Omega(r, \vartheta)$ the equation of momentum in conventional definitions is given by

$$
\frac{\mathrm{d} \boldsymbol{v}}{\mathrm{d} t}+2 \boldsymbol{\Omega} \times \boldsymbol{v}=-\frac{1}{\rho} \nabla p+\boldsymbol{g}+\boldsymbol{r} \times \frac{\mathrm{d} \boldsymbol{\Omega}}{\mathrm{d} t}-\boldsymbol{\Omega} \times(\boldsymbol{\Omega} \times \boldsymbol{r})+\mu_{\mathrm{v}} \nabla^{2} \boldsymbol{v},(1)
$$

where $\mu_{\mathrm{v}}$ is the kinematic/turbulent viscosity coefficient. In the next steps this equation will be simplified keeping the main features of the motion. We consider linear waves without taking into account convective and meridional flows, $\boldsymbol{v}_{\mathbf{0}}=0$, and we suppose that the angular velocity $\Omega$ does not depend on time. For the basic state we have from Eq. (1)

$-\frac{1}{\rho_{0}} \nabla p_{0}+\boldsymbol{g}=\boldsymbol{\Omega} \times(\boldsymbol{\Omega} \times \boldsymbol{r})$.

However, we can exclude practically everywhere in the Sun centrifugal acceleration and consider it as a small 
correction to $\boldsymbol{g}$. Then the spherically symmetric basic state is defined by $\nabla p_{0} \approx \rho_{0} \boldsymbol{g}$. For the linear oscillations we have from Eq. (1):

$$
\begin{aligned}
\frac{\partial \boldsymbol{v}}{\partial t}+2 \boldsymbol{\Omega} \times \boldsymbol{v} & +r \sin \vartheta(\boldsymbol{v} \cdot \nabla \Omega) \boldsymbol{e}_{\boldsymbol{\varphi}}= \\
& -\frac{1}{\rho_{0}} \nabla p^{\prime}+\boldsymbol{g}^{\prime}+\frac{\nabla p_{0}}{\rho_{0}^{2}} \rho^{\prime}+\mu_{\mathrm{v}} \nabla^{2} \boldsymbol{v}
\end{aligned}
$$

Here $\boldsymbol{e}_{\boldsymbol{\varphi}}$ is the unit vector in azimuthal direction, and variables with a prime are Eulerian perturbations. Equation (2) is written in rotating spherical polar coordinates $(r, \vartheta, \varphi)$. It coincides with the equation of motion by Unno et al. (1989) which is derived for an inertial frame, if the operator $\partial / \partial t$ is replaced by $\partial / \partial t+\Omega \partial / \partial \varphi$. To simplify our further discussion we shall use the Cowling approximation, $\boldsymbol{g}^{\prime}=0$, which has a sufficient degree of accuracy for the analysis of short waves.

The next simplification of Eq. (2) is connected with the quasi-rigid rotation of the inner part of the Sun below the convective zone, which is known from the solution of the inverse problem of helioseismology. In this case we can omit the third term of the l.h.s of Eq. (2). Such a restriction of the gradients of $\Omega(r, \vartheta)$ requires to obey the conditions:

$2 \Omega \gg r \frac{\partial \Omega}{\partial r}, 2 \Omega \gg \tan (\vartheta) \frac{\partial \Omega}{\partial \vartheta}$.

To obtain these conditions we used the components of the vector $\boldsymbol{\Omega}$ in spherical polar coordinates, $\boldsymbol{\Omega}=\{\Omega \cos \vartheta$, $-\Omega \sin \vartheta, 0\}$.

The next equations are the mass and energy conservation equations in the standard form:

$\frac{\mathrm{d} \rho}{\mathrm{d} t}+\rho \operatorname{div} \boldsymbol{v}=0$

$c_{\mathrm{v}} \rho \frac{\mathrm{d} T}{\mathrm{~d} t}+p \operatorname{div} \boldsymbol{v}=-\mathcal{L}$,

where: $-\mathcal{L}=Q(\rho, T)-\nabla \cdot \boldsymbol{q}_{\mathrm{R}}-\nabla \cdot\left(K_{0} T^{5 / 2} \nabla T\right)$.

Another form of Eq. (5) is

$\frac{\mathrm{d} p}{\mathrm{~d} t}-c_{\mathrm{s}}^{2} \frac{\mathrm{d} \rho}{\mathrm{d} t}=-(\gamma-1) \mathcal{L}$

Here $\gamma=c_{\mathrm{p}} / c_{\mathrm{v}}, c_{\mathrm{p}}$ and $c_{\mathrm{v}}$ are the specific heats at constant pressure and volume, respectively, $c_{\mathrm{s}}$ is the sound speed, the source function $Q(\rho, T)$ is the sum of nuclear and viscous heat generation rates per unit volume: $\rho\left(\varepsilon_{\mathrm{N}}+\varepsilon_{\mathrm{v}}\right)$, $\mathbf{q}_{\mathrm{R}}$ is the radiative energy flux, $K_{0} T^{5 / 2}$ is the coefficient of electron heat conductivity. In the interior of the Sun we shall neglect viscous heating $\left(\varepsilon_{\mathrm{v}}=0\right)$. For the power of nuclear reactions we have $Q \approx \rho^{2} T^{\alpha}$. In particular for the p-p reaction $Q=\rho \varepsilon_{\mathrm{pp}} \approx 9 \times 10^{-30} \chi_{\mathrm{H}}^{2} \rho^{2} T^{4}$, where $\varepsilon_{\mathrm{pp}}$ is given in $[\mathrm{erg} / \mathrm{g} \mathrm{s}]$ and $\chi_{\mathrm{H}}=0.73$.

In the limit of incompressible fluid, $\mathrm{d} \rho / \mathrm{d} t=0$ or $\operatorname{div} \boldsymbol{v}=0$, it follows from Eq. (6) that the condition $c_{\mathrm{s}}^{2} \rightarrow \infty$ is not needed to satisfy $\mathrm{d} p / \mathrm{d} t \neq 0$. Hence, in a dissipative $(\mathcal{L} \neq 0)$, incompressible fluid sound cannot propagate instantaneously. It means, we cannot use the condition of $c_{\mathrm{s}}^{2} \rightarrow \infty$ to get the incompressible limit for nonadiabatic waves.

Now we will try to simplify the energy loss function $\mathcal{L}$ assuming reasonable approximations for the Sun's interior. We use the formula for the heat conductivity of a fully ionized gas to show that in the Sun radiative transport of energy is more important than that by particle heat conductivity:

$\kappa_{\mathrm{T}}=K_{0} T^{5 / 2} \approx 10^{-6} T^{5 / 2} \mathrm{erg} / \mathrm{s} \mathrm{cm} \mathrm{K}$.

The radiative flux is given by the radiative diffusion equation

$\boldsymbol{q}_{\mathrm{R}}=-\kappa_{\mathrm{R}} \nabla T, \quad \kappa_{\mathrm{R}}=\frac{16 \sigma_{\mathrm{S}}}{3 \chi} T^{3}$,

where $\kappa_{\mathrm{R}}$ is the radiative heat conductivity, $\sigma_{\mathrm{S}}=a_{\mathrm{R}} c / 4=$ $5.67 \times 10^{-5} \mathrm{erg} / \mathrm{cm}^{2} \mathrm{~K}^{4} \mathrm{~s}$ is the Stefan-Boltzmann constant, and $\chi$ is the Rosseland mean opacity. For the opacity we will use Kramers' formula: $\chi=3.68 \times$ $10^{22} \rho^{2} T^{-7 / 2} \mathrm{~cm}^{-1}$. Then, for the radiative conductivity we have

$\kappa_{\mathrm{R}} \approx 8.22 \times 10^{-27} \frac{T^{13 / 2}}{\rho^{2}} \mathrm{erg} / \mathrm{s} \mathrm{cm} \mathrm{K}$.

Now we find that $\kappa_{\mathrm{R}} / \kappa_{\mathrm{T}} \approx 10^{-20} T^{4} / \rho^{2}>10^{3}$. This condition is fulfilled in the whole Sun. That means, we can exclude the heat conductivity term from Eq. (5). The next simplification is connected with the ratio $T^{5 / 2} / \rho$ which is practically not changed over the radius and $\approx 10^{16}$. It means, we can introduce the constant $æ_{0}=$ $8.22 \times 10^{-27} T^{5} / \rho^{2} \approx 8.2 \times 10^{5}$. Then we have

$\kappa_{\mathrm{R}} \approx æ_{0} T^{3 / 2} \mathrm{erg} / \mathrm{s} \mathrm{cm} \mathrm{K}$.

Now for the right-hand side of Eq. (5) we have

$-\mathcal{L} \simeq \frac{2}{5} æ_{0} \nabla^{2} T^{5 / 2}+Q$.

The main non-perturbed energetic state of the Sun is defined by the condition $\mathcal{L}_{0}=0$. In our case this condition is

$Q_{0}=-\frac{2}{5} æ_{0} \frac{\mathrm{d}^{2} T_{0}^{5 / 2}}{\mathrm{~d} r^{2}}$.

\subsection{Choice of the frame of reference}

In the next step we try to get the analytical solutions of the wave equations and to solve the boundary value problem. For this aim we shall investigate the short waves (WKB) approximation for which the effects of curvature are unimportant. It means we can apply the rotating plane-parallel stratification approximation. Such an approach has its advantages and disadvantages. The main advantage is that at the end points of the integration path (center and pole of the Sun) we have no singularity, and this gives a chance to find the solutions analytically. The disadvantages are connected with the following: a) long waves are excluded 
but they are very important, for instance, for the transfer of angular momentum; b) the lost "end" singularities correspond to a real physical behavior of the waves; c) in this approach we get two distinct directions, $\boldsymbol{\Omega}$ and $\boldsymbol{g}$ (taking into account all components of $\boldsymbol{\Omega}$ ). The deviation of the direction of stratification of the plane-parallel fluid layer from the direction of its axis of rotation should lead to additional, physically doubtful results. In geophysical hydrodynamics this problem is solved by applying the " $\beta$ plane" within the frame of the traditional approximation (Pedlosky 1982; Shore 1992), where the component of $\boldsymbol{\Omega}$ parallel to $\boldsymbol{g}$ in the given surface point (local vertical) is retained only in the governing equations. Here we will use the same approach.

Let us take an arbitrary point at the surface of the rotating sphere. The position of this point is defined by its radius $r$, its co-latitude $\vartheta$, and its azimuth angle. We assign to this point a local, left-handed Cartesian system of coordinates $\{x, y, z\}$, where the $\boldsymbol{z}$-axis is directed along the radius (local vertical), the direction of the $\boldsymbol{y}$-axis is meridional (towards the pole), and that of the $\boldsymbol{x}$-axis azimuthal. In this frame of reference $\boldsymbol{\Omega}=\left\{0, \Omega_{y}, \Omega_{z}\right\}=$ $\{0, \Omega \sin \vartheta, \Omega \cos \vartheta\}$. Strictly speaking, the $\boldsymbol{z}$-axis coincides with the rotation axis only at the pole $(\vartheta=0)$. In the case of a homogeneous fluid $\Omega$ is included into the wave equation only in the term

$(\boldsymbol{\Omega} \nabla)^{2}=\Omega_{y}^{2} \frac{\partial^{2}}{\partial y^{2}}+2 \Omega_{y} \Omega_{z} \frac{\partial^{2}}{\partial y \partial z}+\Omega_{z}^{2} \frac{\partial^{2}}{\partial z^{2}}$.

Here we can neglect $\Omega_{y}\left(\Omega_{y}=0\right)$, if the condition $\left|\Omega_{y} \frac{\partial}{\partial y}\right| \ll\left|\Omega_{z} \frac{\partial}{\partial z}\right|$ is fulfilled. This condition is named "traditional approximation". For harmonic motions we have $\frac{\partial}{\partial y}=i k_{y}, \frac{\partial}{\partial z}=i k_{z}$, and the traditional approximation corresponds to $\left|k_{y}\right| \ll\left|k_{z}\right| \cot \vartheta$. If the spatial scale of the wave motion in vertical direction is much smaller than in horizontal direction (at latitudes not close to the equator), we can restrict ourselves to retain only $\Omega_{z}$ in the governing equations. This condition for the traditional approximation remains valid, if a radial stratification is included (Lee \& Saio 1997).

In order to construct the " $\beta$-plane" limit we expand $\Omega_{z}$ around a fixed $\vartheta_{0}\left(\vartheta=\vartheta_{0}+\delta \vartheta\right): \Omega_{z}=\Omega(\vartheta) \cos \vartheta \approx$ $(1+\beta y) \Omega_{0} \cos \vartheta_{0}$. Here $y=R_{\odot} \delta \vartheta, \Omega_{0}=\Omega\left(\vartheta_{0}\right)$, and

$\beta=\left(\frac{1}{\Omega_{0}} \frac{\partial \Omega_{0}}{\partial \vartheta}-\tan \vartheta_{0}\right) \frac{1}{R_{\odot}}$.

Rossby waves are possible only if $\beta \neq 0$. The parameter $\beta$ is a sum of two terms: the second one $\left(\tan \vartheta_{0}\right)$ is due to the geometrical change of the Coriolis parameter with latitude. This term exists always, even if the rotation is rigid. The $r$-modes are connected with this term. The first term in $\beta$ appears if the differential rotation is considered, and the $R$-modes are connected with this term. Close to the pole $\left(\vartheta_{0} \rightarrow 0\right)$ the first term is dominant.

Note that such a " $\beta$ "-limit is applicable also around the equator plane, where the traditional approximation does not fit. The advantage of this limit is the possibility to include $\vartheta$ as a parameter in the Cartesian system.
In this way we use here an inertial Cartesian system of coordinates $(x, y, z)$ in a frame rotating with an angular frequency $\Omega(y, z)$. All non-perturbed model variables are functions of $z$ only, and for the gravity acceleration we have $\boldsymbol{g}=\{0,0,-g(z)\}$. For the observer from the nonrotating frame the elements of fluid are moving due to rotation with a velocity $\boldsymbol{V}_{\mathbf{0}}=\boldsymbol{\Omega} \times \boldsymbol{r}=\{-\Omega y, \Omega x, 0\}$, where in the frame of our approximation $\Omega \approx \Omega_{z}$. $V_{0 x}<0$ means that the $x$-axis is directed opposite to the rotation.

\subsection{Oscillation equations}

For linearization each physical variable $f=f_{0}+f^{\prime}$ is decomposed into a mean term $f_{0}$ and a small fluctuating term $f^{\prime}$. Neglecting terms of higher order than the first one we get our oscillation equations

$$
\begin{aligned}
& \frac{\partial \rho^{\prime}}{\partial t}+v_{z} \frac{\mathrm{d} \rho_{0}}{\mathrm{~d} z}+\rho_{0} \operatorname{div} \boldsymbol{v}=0 \\
& \frac{\partial \boldsymbol{v}}{\partial t}+2 \boldsymbol{\Omega} \times \boldsymbol{v}=-\frac{1}{\rho_{0}} \nabla p^{\prime}+\boldsymbol{g} \frac{\rho^{\prime}}{\rho_{0}}+\mu_{\mathrm{v}} \nabla^{2} \boldsymbol{v} \\
& c_{\mathrm{v}} \rho_{0}\left(\frac{\partial T^{\prime}}{\partial t}+v_{z} \frac{\mathrm{d} T_{0}}{\mathrm{~d} z}\right)+p_{0} \operatorname{div} \boldsymbol{v}=æ_{0} \nabla^{2}\left(T_{0}^{5 / 2} \frac{T^{\prime}}{T_{0}}\right) \\
& +Q_{0}\left(2 \frac{\rho^{\prime}}{\rho_{0}}+\alpha \frac{T^{\prime}}{T_{0}}\right)=-\mathcal{L}^{\prime}
\end{aligned}
$$

For adiabatic oscillations $\mathcal{L}^{\prime}=0$. We approach this regime by setting formally $æ_{0}=0$ (Eq. (9)). But in the nonadiabatic case, which is considered here, $\mathcal{L}^{\prime}$ is the sum of radiative damping and the $\varepsilon$-mechanism terms. Equations (10)(12) must be completed then by the equation of state, $p=p(\rho, T)$. For an ideal gas we have

$\frac{p^{\prime}}{p_{0}}=\frac{\rho^{\prime}}{\rho_{0}}+\frac{T^{\prime}}{T_{0}}$

For the ideal gas $p_{0}=R_{\mathrm{g}} \rho_{0} T_{0} / \mu_{\mathrm{m}}$, where $R_{\mathrm{g}}$ is the gas constant, $\mu_{\mathrm{m}}$ is the molecular weight, and the squared adiabatic sound speed $c_{\mathrm{s}}^{2}=\gamma c^{2}$ is defined by the squared isothermal sound speed $c^{2}=p_{0} / \rho_{0}$.

We define

$$
\begin{aligned}
& \tilde{Q}_{0}=\frac{Q_{0}}{c_{\mathrm{v}} \rho_{0} T_{0}}, \quad \tilde{\aleph}_{0}=\frac{æ_{0}}{c_{\mathrm{v}} \rho_{0} T_{0}}, \quad N^{2}=g\left(\frac{1}{\gamma} æ_{\mathrm{p}}-æ_{\rho}\right), \\
& æ_{\mathrm{T}}=\frac{1}{T_{0}} \frac{\mathrm{d} T_{0}}{\mathrm{~d} z}, \quad æ_{\rho}=\frac{1}{\rho_{0}} \frac{\mathrm{d} \rho_{0}}{\mathrm{~d} z} \\
& æ_{\mathrm{p}}=\frac{1}{p_{0}} \frac{\mathrm{d} p_{0}}{\mathrm{~d} z}=æ_{\rho}+æ_{\mathrm{T}}=-\frac{g}{c^{2}} .
\end{aligned}
$$

Here $N^{2}(z)$ is the squared Brunt-Väisälä frequency. $æ_{\mathrm{p}}, æ_{\rho}$, and $æ_{\mathrm{T}}$ are the reciprocal pressure, density, and temperature inhomogeneity scales, respectively, and $\tilde{Q}_{0}$ is the reciprocal of the characteristic Kelvin time-scale - a deviation from the thermal balance of the star is restored during this time. As the coefficients of Eqs. (10)-(13) are independent of the time $t$ and of the space coordinate $x$ we can set

$$
\frac{\partial}{\partial t}=-i \omega, \frac{\partial}{\partial x}=i k_{x}
$$


Now we exclude $\rho^{\prime}$ from the system of Eqs. (10)-(13) and have

$-i \omega\left(\frac{p^{\prime}}{p_{0}}-\frac{T^{\prime}}{T_{0}}\right)+æ_{\rho} v_{z}+u=0$,

$-i k_{x} c^{2} \frac{p^{\prime}}{p_{0}}+2 \Omega v_{y}+\check{D} v_{x}=0$

$-c^{2} \frac{\partial}{\partial y} \frac{p^{\prime}}{p_{0}}-2 \Omega v_{x}+\check{D} v_{y}=0$

$-c^{2} \frac{\partial}{\partial z} \frac{p^{\prime}}{p_{0}}+g \frac{T^{\prime}}{T_{0}}+\check{D} v_{z}=0$

$-\left(i \omega+2 \tilde{Q}_{0}\right) \frac{p^{\prime}}{p_{0}}+æ_{\mathrm{p}} v_{z}+\gamma u=\check{f}$,

$u=\operatorname{div} \boldsymbol{v}=i k_{x} v_{x}+\frac{\partial v_{y}}{\partial y}+\frac{\partial v_{z}}{\partial z}$,

where the operators $\check{D}$ and $\check{f}$ are defined as

$\check{D}=i \omega+\mu_{\mathrm{v}} \nabla^{2}, \check{f}=(\alpha-2) \tilde{Q}_{0} \frac{T^{\prime}}{T_{0}}+\tilde{œ}_{0} \nabla^{2}\left(T_{0}^{5 / 2} \frac{T^{\prime}}{T_{0}}\right)$

and the relation $p_{0} /\left(c_{\mathrm{v}} \rho_{0} T_{0}\right)=\gamma-1$ is used. In the next steps the viscosity appears in the coefficients of the equations in the form $\frac{c^{2}}{\omega}+\mu_{\mathrm{v}} \cdot O(1)$. For a fully ionized plasma the kinematic viscosity is $\mu_{\mathrm{v}} \approx 10^{-16} T^{5 / 2} / \rho\left[\mathrm{cm}^{2} / \mathrm{s}\right]$. For the solar situation $\mu_{\mathrm{v}} \approx O(1)$, if we do not take into account the turbulent viscosity. As we are interested in very low frequencies the condition $\frac{c^{2}}{\omega} \gg \mu_{\mathrm{v}}$ is valid. Then we can put $\check{D} \simeq i \omega$ (non-viscous case). Now Eqs. (16)-(17) define the horizontal components of velocity and hence its two-dimensional divergence $\operatorname{div}_{\perp} \boldsymbol{v}$ :

$v_{x}=-\frac{k_{x} c^{2}}{\omega} \frac{\sigma^{2}}{1-\sigma^{2}}\left(1+\frac{1}{\sigma k_{x}} \frac{\partial}{\partial y}\right) \frac{p^{\prime}}{p_{0}}$,

$v_{y}=i \frac{k_{x} c^{2}}{\omega} \frac{\sigma}{1-\sigma^{2}}\left(1+\frac{\sigma}{k_{x}} \frac{\partial}{\partial y}\right) \frac{p^{\prime}}{p_{0}}$,

$\operatorname{div}_{\perp} \boldsymbol{v}=c^{2} i k_{x} \frac{\sigma^{\prime}}{\omega} \check{M} \frac{p^{\prime}}{p_{0}}$,

where

$\check{M}=1+\frac{2 \sigma}{k_{x}} \frac{\partial}{\partial y}+\frac{\sigma^{2}}{k_{x} \sigma^{\prime}}\left(\frac{\partial^{2}}{\partial y^{2}}-k_{x}^{2}\right), \sigma=\frac{\omega}{2 \Omega}, \sigma^{\prime}=\frac{\partial \sigma}{\partial y}$.

Excluding $u=\operatorname{div}_{\perp} \boldsymbol{v}+\partial v_{z} / \partial z$ by using Eq. (15) we have the system of equations

$i \omega v_{z}=c^{2} \frac{\partial}{\partial z} \frac{p^{\prime}}{p_{0}}-g \frac{T^{\prime}}{T_{0}}$

$\frac{T^{\prime}}{T_{0}}-\check{s}(y, z) \frac{p^{\prime}}{p_{0}}+\frac{1}{i \omega}\left(æ_{\rho}+\frac{\partial}{\partial z}\right) v_{z}=0$,

$q \frac{p^{\prime}}{p_{0}}+\frac{1}{i \omega} \frac{N^{2}}{g} v_{z}=\frac{T^{\prime}}{T_{0}}+\frac{1}{\gamma_{*}} \check{f}$,

where the dimensionless quantites are

$\check{s}=1-c^{2} k_{x} \frac{\sigma^{\prime}}{\omega^{2}} \check{M}, \gamma_{*}=\gamma i \omega, q=\frac{\gamma-1}{\gamma}-\frac{2 \tilde{Q}_{0}}{\gamma_{*}}$.
Now we use the condition $\sigma \ll 1$ and receive

$v_{x}=-\frac{c^{2}}{2 \Omega} \frac{\partial}{\partial y} \frac{p^{\prime}}{p_{0}}$

$v_{y}=\frac{c^{2}}{2 \Omega} i k_{x} \frac{p^{\prime}}{p_{0}}$.

In the case of rigid rotation, $\frac{\partial \Omega}{\partial y}=0$, it follows immediately from Eqs. (29), (30) that $\operatorname{div}_{\perp} \boldsymbol{v}=0$. It means, that the incompressible case $(\operatorname{div} \boldsymbol{v}=0)$ for which everywhere $v_{z}=0$ (from the boundary condition at the center) is not so interesting for astrophysical situations. In this case $\rho^{\prime}=0$ and the equation of heat conductivity, Eq. (12), is separated from the equation of motion. In geophysical situations just this case is interesting, when geostrophic eddies are investigated. For our task waves with $v_{z} \neq 0$ are more important.

Our next step is to separate the $z$ and $y$ dependence of the variables in the governing equations to have finally one ordinary differential equation. In the general case such a separation is not possible, and we consider here the very simple case when the function $\frac{\partial}{\partial y}\left(\frac{1}{\Omega}\right)$ is independent of $y$. Only in this case it is possible to separate the equations with respect to the variables $y$ and $z$ and we can write $\frac{\partial^{2}}{\partial y^{2}}=-k_{y}^{2}$. Here $k_{y}$ should be determined from the boundary conditions, and a complex $k_{y}$ is not excluded. In this way the system of partial differential equations in the plane-parallel approximation is reduced to ordinary differential equations. Now we assume the following formula for the rotation profile

$\Omega(y, z)=\frac{\tilde{\Omega}(z)}{1+\beta y / R_{\odot}}$,

where $\beta>0$. This profile reveals that the rotation rate decreases at the pole. If $y \rightarrow \infty$ then $\Omega \rightarrow 0$. Helioseismology inversions predict $\Omega(\vartheta)$ in the convective zone which is almost the same as that at the surface $(\beta \approx O(1))$, but below the bottom of the convective zone (at the tachocline) rotation is close to a solid-body rotation $(\beta \ll 1)$. As $y_{*}=y / R_{\odot} \leq 1$, in the deepest layers of the Sun we can assume the rotation rate $\Omega(y, z) \approx \tilde{\Omega}(z)\left(1-\beta y_{*}\right)$, where $\tilde{\Omega}(z) \approx O\left(\Omega_{\odot}\right)$. Then we have in Eq. $(28)$

$\check{M} \approx M=1+\frac{\omega k_{\perp}^{2}}{k_{x} 2 \Omega^{\prime}(y)}, \check{s}=s \approx 1-M \frac{c^{2} k_{x}}{2 \omega} \frac{\partial}{\partial y} \frac{1}{\Omega}$,

where $k_{\perp}^{2}=k_{x}^{2}+k_{y}^{2}$.

Our next step is to derive one differential equation for the temperature perturbations. The variable $v_{z}$ is easily excluded from Eqs. (25)-(27). Then we get for the pressure perturbations

$$
\begin{aligned}
a \frac{p^{\prime}}{p_{0}}= & \frac{T^{\prime}}{T_{0}}+\left(\frac{\omega}{c d}\right)^{2} q\left[\left(1-\frac{N^{2}}{\omega^{2}}\right) \frac{T^{\prime}}{T_{0}}+\frac{\check{f}}{\gamma_{*}}\right] \\
& +\left(æ_{\rho}+\frac{\partial}{\partial z}\right)\left(\frac{T^{\prime}}{T_{0}}+\frac{\check{f}}{\gamma_{*}}\right) \frac{1}{d(z)},
\end{aligned}
$$

where

$a=s+\left(\frac{\omega q}{c d}\right)^{2}+\left(æ_{\rho}+\frac{\partial}{\partial z}\right) \frac{q}{d}, d=\frac{N^{2}}{g}=\frac{æ_{\mathrm{p}}}{\gamma}-æ_{\rho}$. 
The parameter $d$ is positive in the solar radiative interior, $d>0$, while we have $d \leq 0$ in the convective zone. The pressure perturbations (Eq. (33)) have a singularity at $a=$ 0 . However, it will be shown below that this singularity is removable.

Introducing a new dependent variable

$\Theta=\kappa \frac{T^{\prime}}{T_{0}}, \kappa=\left(\frac{T_{0}}{T_{00}}\right)^{5 / 2}, T_{00}=$ const.

we receive the final equation of fourth order

$\Theta^{\prime \prime \prime \prime}+A_{3} \Theta^{\prime \prime \prime}+A_{2} \Theta^{\prime \prime}+A_{1} \Theta^{\prime}+A_{0} \Theta=0$,

where $\Theta^{\prime}=\mathrm{d} \Theta / \mathrm{d} z, \varepsilon_{0}=\tilde{\mathfrak{x}}_{0} T_{0}^{5 / 2} / \gamma_{*}$ and

$$
\begin{aligned}
& A_{0}=a_{1} a_{2}+a_{2}^{\prime}-\frac{a \omega^{2}}{\varepsilon_{0} c^{2}}\left(\frac{N^{2}}{\omega^{2}}-\varepsilon_{0} a_{3}\right), \\
& A_{1}=a_{1} a_{3}+a_{2}+a_{3}^{\prime}, \\
& A_{2}=a_{3}-a_{1} b_{2}-b_{2}^{\prime}, \quad A_{3}=-\frac{\mathrm{d}}{\mathrm{d} z} \ln \left(p_{0} T_{0} a d^{2}\right), \\
& -a_{1}=\frac{a^{\prime}}{a}+\frac{d^{\prime}}{d}+æ_{\mathrm{p}}+\frac{q \omega^{2}}{d c^{2}}, \\
& a_{2}=\frac{1}{\varepsilon_{0}}\left(b_{1}-\frac{5}{2} æ_{\mathrm{T}}\right)+æ_{1}^{\prime}+b_{2} \tilde{k_{\perp}^{2}}, \\
& a_{3}=\frac{1}{\varepsilon_{0}}-\tilde{k_{\perp}^{2}}, \quad \tilde{k_{\perp}^{2}}=k_{x}^{2}+k_{y}^{2}-æ_{1}=k_{\perp}^{2}-æ_{1}, \\
& b_{1}=\frac{æ_{\mathrm{p}}}{\gamma}-\frac{d^{\prime}}{d}-\frac{q \omega^{2}}{d c^{2}}\left(\frac{N^{2}}{\omega^{2}}-1\right), b_{2}=æ_{\mathrm{T}}+\frac{d^{\prime}}{d}-\frac{q \omega^{2}}{d c^{2}}, \\
& æ_{1}=\frac{2}{5}(2-\alpha) \frac{\kappa^{\prime \prime}}{\kappa}, \quad q=\frac{\gamma-1}{\gamma}+\frac{4}{5} \varepsilon_{0} \frac{\kappa^{\prime \prime}}{\kappa} .
\end{aligned}
$$

In order to solve Eq. (36) we normalize it to get a dimensionless equation. For this purpose we normalize the radial distance to the solar radius: $z \Rightarrow z / R_{\odot}$ and get finally the following equation

$$
\begin{aligned}
& \varepsilon \Theta^{\prime \prime \prime \prime}+\varepsilon \varphi_{3} \Theta^{\prime \prime \prime}+\left(1+\varepsilon \varphi_{2}\right) \Theta^{\prime \prime}+ \\
& +\left(\psi_{1}+\varepsilon \varphi_{1}\right) \Theta^{\prime}+\left(\psi_{0}+\varepsilon \varphi_{0}\right) \Theta=0,
\end{aligned}
$$

where we kept the notation $\tilde{k_{\perp}^{2}} \Rightarrow R_{\odot}^{2} \tilde{k_{\perp}^{2}}$, the inverse scale heights $æ_{\mathrm{p}, \mathrm{T}}$ are defined as above (below Eq. (13)) but with a normalized $z$, and

$$
\begin{aligned}
& \psi_{0}=-a \tilde{\omega}^{2}\left(N^{2} / \omega^{2}-1+\varepsilon \tilde{k_{\perp}^{2}}\right)+b_{1}\left(a_{1}+æ_{\mathrm{T}}+b_{1}^{\prime} / b_{1}\right), \\
& \psi_{1}=-\frac{\mathrm{d}}{\mathrm{d} z} \ln \left(T_{0}^{3 / 2} a d^{2}\right), \quad \varphi_{3}=-\frac{\mathrm{d}}{\mathrm{d} z} \ln \left(p_{0} T_{0} a d^{2}\right), \\
& \varphi_{0}=a_{1}\left(æ_{1}^{\prime}+b_{2} \tilde{k}_{\perp}^{2}\right)+\left(æ_{1}^{\prime}+b_{2} \tilde{k}_{\perp}^{2}\right)^{\prime}, \\
& \varphi_{1}=-\tilde{k_{\perp}^{2}} \varphi_{3}+\frac{4}{5} æ_{\mathrm{p}} \frac{\kappa^{\prime \prime}}{\kappa}+2 æ_{1}^{\prime} \text {, } \\
& -\varphi_{2}=\tilde{k_{\perp}^{2}}+a_{1} b_{2}+b_{2}^{\prime}-\tilde{\omega}^{2} a \\
& -a_{1}=\frac{a^{\prime}}{a}+\frac{d^{\prime}}{d}+æ_{\mathrm{p}}+\tilde{\omega}^{2} \frac{q}{d}, \quad q=\frac{\gamma-1}{\gamma}+\frac{4}{5} \varepsilon \frac{\kappa^{\prime \prime}}{\kappa}, \\
& b_{1}=\frac{æ_{\mathrm{p}}}{\gamma}-\frac{5}{2} æ_{\mathrm{T}}-\frac{d^{\prime}}{d}-\tilde{\omega}^{2} \frac{q}{d}\left(\frac{N^{2}}{\omega^{2}}-1\right) \text {, } \\
& b_{2}=æ_{\mathrm{T}}+\frac{d^{\prime}}{d}-\tilde{\omega}^{2} \frac{q}{d}, \quad M=1-\frac{\omega}{2 \tilde{\Omega}} \frac{\bar{k}_{\perp}^{2}}{\beta \overline{k_{x}}},
\end{aligned}
$$

$$
\begin{aligned}
& a=1-\Lambda+\left(\tilde{\omega} \frac{q}{d}\right)^{2}+\left(æ_{\rho}+\frac{\partial}{\partial z}\right) \frac{q}{d} \\
& \varepsilon=\frac{\varepsilon_{0}}{R_{\odot}^{2}}=\frac{æ_{0} T_{0}^{5 / 2}}{c_{\mathrm{v}} \rho_{0} T_{0}} \frac{1}{\gamma_{*} R_{\odot}^{2}}=\frac{1}{\gamma_{*} R_{\odot}^{2}} \frac{\kappa_{\mathrm{R}}}{c_{\mathrm{v}} \rho_{0}} \\
& \bar{k}_{x, \perp}=k_{x, \perp} R_{\odot}, \quad \tilde{\omega}=\omega R_{\odot} / c, \quad \Lambda=\beta \frac{k_{x} c^{2} M}{2 \tilde{\Omega} \omega R_{\odot}}
\end{aligned}
$$

Recall that $\gamma_{*}=\gamma i \omega$. Here the main parameter is $\Lambda$, that includes the rotation rate gradient ( $\beta=$ const.).

Because we are interested in very low frequency oscillations with periods of $1-20$ years, we take $\omega \approx 10^{-8} \mathrm{~s}^{-1}$, $\frac{T_{0}^{5 / 2}}{\rho_{0}} \approx$ const. $=10^{16}, c_{\mathrm{v}} \approx 2 \times 10^{8}, æ_{0} \approx 8 \times 10^{5}$, and we have a small parameter for our task

$\varepsilon(z) \approx 10^{-8} \frac{T_{\mathrm{c}}}{T_{0}(z)}$.

Here $T_{\mathrm{c}}$ is the temperature of the solar center. For the whole Sun this parameter $\varepsilon$ is changed in the interval $10^{-8} \leq \varepsilon \leq 10^{-5}$. $\varepsilon$ characterizes the degree of nonadiabaticity of the waves, which is defined as the ratio of the wave period to the Kelvin time.

\section{Adiabatic case}

For idealized adiabatic waves $(\varepsilon=0)$ we have a second order equation,

$\Theta^{\prime \prime}+\psi_{1} \Theta^{\prime}+\psi_{0} \Theta=0$.

Introducting a new variable it may be written in standard form

$\Theta=Y \sqrt{T_{0}^{3 / 2} a d^{2}}, \quad Y^{\prime \prime}(z)+I(z) Y=0$,

where

$I=\psi_{0}-\frac{1}{4}\left[\frac{\mathrm{d}}{\mathrm{d} z} \ln \left(T_{0}^{3 / 2} a d^{2}\right)\right]^{2}+\frac{1}{2} \frac{d^{2}}{d z^{2}} \ln \left(T_{0}^{3 / 2} a d^{2}\right)$.

$\psi_{0}$ and $a$ are defined by Eqs. $\left(37^{\prime}\right)$ and $d=R_{\odot} N^{2} / g$. The behavior of the function $I(z)$ gives a possibility to analyze qualitatively the waves in the solar interior and the boundaries from which the waves are reflected and become trapped. If $I>0$ we have oscillating solutions and if $I \leq 0$ the waves are exponentially decreasing (evanescent) with $z$. Now we shall breafly discuss the incompressible and compressible cases.

As we consider adiabatic waves, the transition to the incompressible case can be done by $c^{2} \rightarrow \infty$. In this case $æ_{\mathrm{p}} \rightarrow 0, d \rightarrow-æ_{\rho},|\Lambda| \rightarrow \infty$ and instead of Eq. (41) we obtain

$I \approx \beta \bar{k}_{x} \frac{\omega}{2 \tilde{\Omega}}\left(\frac{N^{2}}{\omega^{2}}-1\right)-\frac{5}{2} æ_{\mathrm{T}} æ_{\Omega}-\frac{1}{4}\left(\frac{5}{2} æ_{\mathrm{T}}-æ_{\Omega}\right)^{2}$,

where $\bar{k}_{x}=k_{x} R_{\odot}$ and $æ_{\Omega}=\mathrm{d} \ln \tilde{\Omega} / \mathrm{d} z$. Here there are three possibilities:

1) absolute rigid body rotation, $\beta=æ_{\Omega}=0$. In this case $I<0$ and we have no cavity solutions; 
2 ) rigid rotation with respect to latitude $(\beta=0)$, but "vertical" differential rotation with respect to the radius $\left(æ_{\Omega} \neq 0\right)$. It follows from Eq. (42) that in this case for oscillating solutions we must have $æ_{\Omega}>0$ as $\mathfrak{x}_{\mathrm{T}}<0$ is obeyed in the inner part of the Sun. It means that solar rotation must have a decreasing speed toward the center $(\mathrm{d} \Omega / \mathrm{d} z>0)$. Then for $I>0$ the condition $10\left|æ_{\mathrm{T}}\right| æ_{\Omega}>\left(5 æ_{\mathrm{T}} / 2-æ_{\Omega}\right)^{2}$ must be fulfilled;

3) The most realistic case for the Sun is a differential rotation in both directions $(\beta \neq 0$ and $\mathrm{d} \Omega / \mathrm{d} z \neq 0)$. In this case we have various chances to get trapped waves. We are interested in oscillations with $\omega \approx 10^{-8} \times \mathrm{s}^{-1}$ (years) and $\bar{k}_{x} \approx 10^{2}\left(\lambda \approx 3 \times 10^{4} \mathrm{~km}\right.$, e.g. sunspots $)$. For solar conditions $\left(\Omega_{\odot} \approx 2.86 \times 10^{-6} \mathrm{~s}^{-1}\right.$ and $\left.N_{\max }^{2} \approx 6 \times 10^{-6} \mathrm{~s}^{-2}\right)$ we have $\bar{k}_{x} \omega / 2 \Omega \approx 1$ and $N_{\max }^{2} / \omega^{2} \approx 10^{10}$. So the dominant term in Eq. (42) is the first one. To have an oscillating solution $(I>0)$ it is sufficient to have a very slow latitudinal differential rotation, $\beta \geq 10^{-8} \approx O\left(\omega\left[\mathrm{s}^{-1}\right]\right)$.

For waves running in opposite direction to rotation in the azimuth $\left(\beta \bar{k}_{x}>0\right)$, the cavities (trapped wave area) can form between the bottom of the convective zone and almost the center of the Sun as well as in the outer part of the Sun where $N^{2}>0$.

Waves propagating parallel to rotation may be trapped only in the convective zone $\left(N^{2}<0\right)$. To the outer and inner sides from the convective zone the amplitude of these waves decrease exponentially.

For the incompressible case it is easy to solve the eigenvalue problem of the cavity oscillations, because Eq. (42) has no singularity. Such a task has been solved by Oraevsky \& Dzhalilov (1997). However, in the nonadiabatic case we cannot apply the limit $c^{2} \rightarrow \infty$. Therefore we have to investigate the more complicated compressible case.

To investigate the function $I(z)$ given by Eq. (41) in a compressible plasma we need the orders of the quantities entering the function $I(z)$. To estimate these values let us consider a linear profile of temperature, $T_{0} \approx T_{\mathrm{c}}\left(1-\beta_{\mathrm{T}} z\right)$, where the gradient $\beta_{\mathrm{T}}=1-T_{\text {eff }} / T_{\mathrm{c}} \approx 1, T_{\text {eff }}$ is the effective temperature. Then we have a limit for the parameter $æ_{\mathrm{T}}$ from the center $(z=0)$ up to the surface $(z=1): 1 \leq-\mathfrak{T}_{\mathrm{T}} \leq 10^{3}$. The other parameters have the same order, $æ_{\mathrm{p}, \rho} \approx O\left(æ_{\mathrm{T}}\right)$. Then we get also: $\mathfrak{x}_{\mathrm{T}}^{\prime} \approx-\mathfrak{x}_{\mathrm{T}}^{2}, æ_{\mathrm{p}}^{\prime} \approx æ_{\mathrm{p}} æ_{\mathrm{T}}, æ_{\rho}^{\prime} \approx æ_{\mathrm{T}}\left(æ_{\mathrm{p}}+æ_{\mathrm{T}}\right)$. Now we can estimate the sign behavior of $I(z)$. We shall consider more characteristic places of the Sun. In the following the condition $\beta \bar{k}_{x}>0$ will be supposed.

At the center, where $z \rightarrow 0, g \rightarrow 0, N^{2} \rightarrow 0, æ_{\mathrm{p}} \rightarrow$ $0, c \approx 350 \mathrm{~km} \mathrm{~s}^{-1}$, if $\Lambda>1$ we have

$I \approx-\beta \bar{k}_{x} \frac{\omega}{2 \tilde{\Omega}}-\frac{3}{2} æ_{\Omega}-\frac{1}{4}\left(\frac{1}{2} æ_{\mathrm{T}}-æ_{\Omega}\right)^{2}-\frac{1}{2} æ_{\mathrm{T}}^{2}<0$.

In the middle part of the Sun (between the edge of the core and the bottom of the convective zone), where $N^{2} \approx N_{\max }^{2}$ and $x_{\rho} \approx$ const $\approx-10$, the dominant term in the function $I(z)$ is the first term of function $\psi_{0}$. Hence we have $I>0$ such as in the incompressible case.

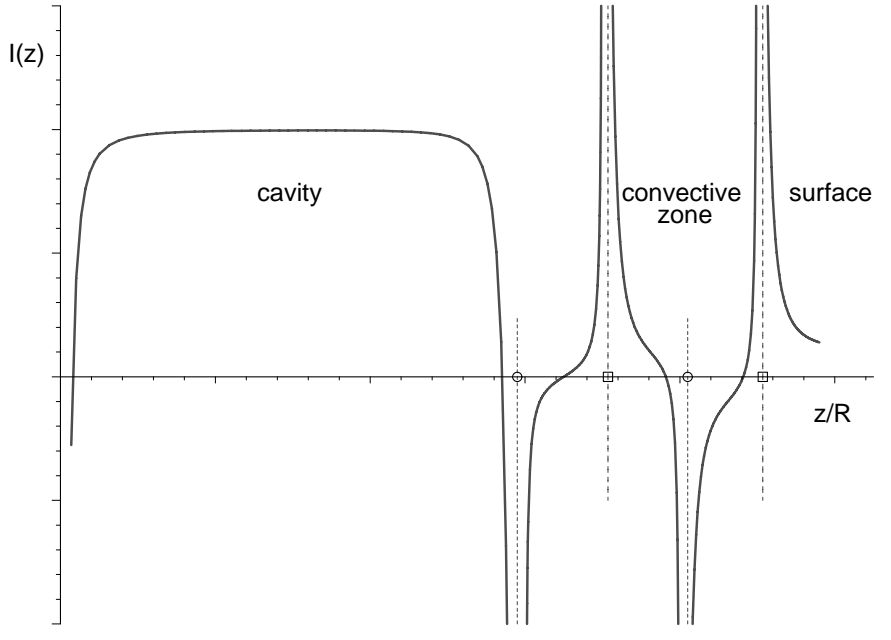

Fig. 1. Scheme of the dependence of the wave potential $I(z)$ defined by Eq. (41) on the distance normalized to the solar radius. Zeros of this function $I(z)=0$ (turning points) divide the wave zone into transparent (cavity) and opaque (tunnel) parts. In the upper part of the Sun there are singular points of the function $I(z)$ which are located between the turning points: the circles correspond to $a(z)=0$ and the boxes correspond to $N^{2}(z)=0$ (boundaries of the convective zone). The narrow area at the bottom of the convective zone between circles and boxes is the tachocline. The main internal cavity comprises the whole radiative interior as the convective zone becomes opaque for the waves.

The area around the convective zone is more complicated. The function $I(z)$ has singularities at the points where $d=0$ and $a=0 ; d$ is connected with the BruntVäisälä frequency and $a$ is defined mainly by the rotation gradient, Eqs. $\left(37^{\prime}\right)$. The two points of $d=0$ correspond to the bottom and the upper boundary of the convective zone. The function $a(z)$ is defined mainly by two terms

$a \approx-\Lambda-\frac{\gamma-1}{\gamma} \frac{d^{\prime}}{d^{2}}$

It is clear that as $d^{\prime}<0$ at the lower boundary of the convective zone, the two zeros of $a(z)$ are located around this boundary where $d=0$. We can easily conclude that

$\lim _{a \rightarrow 0} I(z) \approx-\frac{3}{4}\left(\frac{a^{\prime}}{a}\right)^{2} \rightarrow-\infty$

$\lim _{d \rightarrow 0} I(z) \approx 2\left(\frac{d^{\prime}}{d}\right)^{2} \rightarrow+\infty$.

In the convective zone $\left(N^{2}<0\right)$ we have $I<0$. At the surface of the Sun we have again $N^{2}>\omega^{2}$ and hence $I>0$.

Thus if we approach the convective zone from below there exists a sequence of special points: four times the function $I(z)$ crosses zero (turning points), and between these zeros the singular points are placed (see Fig. 1). The turning points are determined practically by the condition of $N^{2}(z)-\omega^{2}=0$ and the singularities by the conditions $a=0$ (circles in Fig. 1) and $N^{2}=0$ (boxes in Fig. 1). Due 
to the very low frequency and the sharp decrease of $N^{2}(z)$ the turning point of the main inner cavity $z_{\mathrm{t}}$ is very close to the first singular point where $a=0$.

In this way for waves with $k_{x}>0$ a main large internal cavity is placed practically between the center and the bottom of the tachocline. The solar atmosphere is a wave-propagating zone. Between the inner cavity and the solar surface a dark convective tunnel is placed; a very narrow wave-trapping zone around the bottom of convective zone is also possible. It is clear that a tunneling of waves across the magnetized and turbulent convective area to the surface is probably possible. For waves with $k_{x}<0$ the convective zone becomes a cavity. In this case the waves cannot be propagating at the solar surface.

Not all singularities of the wave potential $I(z)$ are singular levels of the physical variables. Around the point $z_{\mathrm{d}}$ where $d\left(z_{\mathrm{d}}\right)=0$ we can write $d \approx d^{\prime}\left(z-z_{\mathrm{d}}\right)$. Then we have the equation $x^{2} Y^{\prime \prime}+2 Y=0$, where $x=z-z_{\mathrm{d}}$. The solutions of this equation are $Y_{1,2}=\sqrt{x} x^{ \pm i \sqrt{7} / 2}$. As $a \sim d^{-2}$ we get from Eqs. $(33)+(40)$ that $p^{\prime} / p_{0} \sim \Theta \sim Y_{1,2} \rightarrow 0$ if $z \rightarrow z_{\mathrm{d}}$. It means, that the boundaries of the convective zone are not singular levels for the initial physical variables.

Another situation exists at the point $z=z_{\mathrm{a}}$ where $a=$ 0 . If we denote now $x$ as $x=z-z_{\mathrm{a}}$, our Eq. (40) around the point $x \approx 0$ is $x^{2} Y^{\prime \prime}-Y 3 / 4=0$, the solutions of which are $Y_{1}=x^{3 / 2}, Y_{2}=x^{-1 / 2}$. Hence, for these solutions we have $\Theta_{1} \approx x^{2}, \Theta_{2} \approx$ const. Then we get from Eq. (33) that the second solution in $p^{\prime} / p_{0}$ diverges at $a=0$.

There exist methods to construct asymptotic solutions of differential equations of second order with a singular turning point. However, we are now returning to our fourth order Eq. (37) for two reasons: our intent is to consider the instability problem of the eigenmodes, and consequently in the complex $\omega$ plane the singularity at $a(z, \omega)=0$ is removed from the real $z$-axis.

\section{Asymptotic solutions}

The existence of the small parameter $\varepsilon$ in Eq. (37) allows us to apply asymptotic methods to solve this equation. Here we shall construct the inner cavity solutions only. As it has been discussed above the coefficients of Eq. (37) vary over a wide range. Very crudely, we have the following estimates from the center of the Sun to the bottom of the convective zone: $\varepsilon \approx O\left(10^{-8}-10^{-5}\right), æ_{\mathrm{T}} \approx O(1-$ $\left.10^{3}\right), \varphi_{3} \approx O\left(æ_{\mathrm{T}}\right), \varphi_{2} \approx O\left(æ_{\mathrm{T}}^{2}\right), \varphi_{1} \approx O\left(æ_{\mathrm{T}}^{3}\right), \varphi_{0} \approx$ $O\left(æ_{\mathrm{T}}^{4}\right), \psi_{1} \approx O\left(æ_{\mathrm{T}}\right)$, and $\psi_{0} \approx O\left(æ_{\mathrm{T}}^{2}\right)$. Assuming that $\varepsilon æ_{\mathrm{T}}^{2} \approx O\left(10^{-8}-10\right)$ we can separate the variable part of $\varepsilon$ as $\varepsilon=\tilde{\varepsilon} T_{\mathrm{c}} / T_{0}(z)$ and rewrite Eq. (37) in a convenient form:

$$
\begin{aligned}
& \tilde{\varepsilon}\left(\Theta^{\prime \prime \prime \prime}+\Phi_{3} \Theta^{\prime \prime \prime}\right)+\Phi_{2} \Theta^{\prime \prime}+\Phi_{1} \Theta^{\prime}+\Phi_{0} \Theta=0, \\
& \Phi_{0}=\frac{T_{0}}{T_{\mathrm{c}}}\left(\psi_{0}+\varepsilon \varphi_{0}\right), \Phi_{1}=\frac{T_{0}}{T_{\mathrm{c}}}\left(\psi_{1}+\varepsilon \varphi_{1}\right), \\
& \Phi_{2}=\frac{T_{0}}{T_{\mathrm{c}}}\left(1+\varepsilon \varphi_{2}\right), \Phi_{3}=\varphi_{3} .
\end{aligned}
$$

The general solution of this equation describes the slow and fast oscillating motions coupled with each other (quasi-adiabatic and dissipative modes). Every of these solutions is searched in a different way.

\subsection{Dissipative modes}

The solutions corresponding to the dissipative modes are searched for in the form:

$\Theta=B_{0}(z)\left(1+\tilde{\varepsilon}^{1 / 2} B_{1}+\tilde{\varepsilon} B_{2}+\cdots\right) \mathrm{e}^{\tilde{\varepsilon}^{-1 / 2} \int^{z} S(z) \mathrm{d} z}$

where $S(z)$ and $B_{0,1,2, \ldots}(z)$ are unknown regular functions. Inserting the formal solution (46) into Eq. (45) we may find all the unknown functions by the usual methods. In particular we have

$$
\begin{aligned}
S^{2}= & -\Phi_{2}, \quad S B_{0}=\Phi_{2}^{-3 / 4} \mathrm{e}^{\frac{1}{2} \int^{z} F_{0} \mathrm{~d} z} \\
B_{1}= & \frac{1}{2} \int^{z} \frac{F_{1}}{S B_{0} \Phi_{2}} \mathrm{~d} z \\
F_{0}= & \frac{\Phi_{1}}{\Phi_{2}}-\Phi_{3}=\frac{\psi_{1}+\varepsilon \varphi_{1}}{1+\varepsilon \varphi_{2}}-\varphi_{3}, \\
F_{1}= & S\left(2 S B_{0}^{\prime \prime}+3 S^{\prime} B_{0}^{\prime}+S^{\prime \prime} B_{0}\right)+3\left[S\left(S B_{0}\right)^{\prime}\right]^{\prime} \\
& +3 \Phi_{3} S\left(S B_{0}\right)^{\prime}+\Phi_{1} B_{0}^{\prime}+\Phi_{0} B_{0} .
\end{aligned}
$$

In the derivation of these functions we have used the obvious condition $\tilde{\varepsilon}^{-1 / 2} S B_{0} \gg B_{0}^{\prime}$. It may easily be shown that at the boundaries of the convective zone where $d=0$ the solution (46) is limited. To solve the eigenvalue problem we can restrict ourselves to the main branch of the asymptotic solutions of (46):

$$
\Theta \approx\left(\frac{T_{\mathrm{c}}}{T_{0}} \frac{1}{1+\varepsilon \varphi_{2}}\right)^{5 / 4} \mathrm{e}^{\frac{1}{2} \int^{z} F_{0} \mathrm{~d} z} \mathrm{e}^{ \pm i \int^{z}\left(\frac{1}{\varepsilon}+\varphi_{2}\right)^{1 / 2} \mathrm{~d} z}
$$

In order to get the final form of this solution let us consider the extreme case $\varepsilon \rightarrow 0$. Then we have

$$
\Theta \approx \frac{\sqrt{\rho_{0}}}{T_{0}} \exp \left( \pm i \int^{z} \frac{\mathrm{d} z}{\sqrt{\varepsilon}}\right)
$$

where $1 / \varepsilon=i /|\varepsilon|$ and $1 / \sqrt{\varepsilon}= \pm(1+i) / \sqrt{2|\varepsilon|}$. It is clear that the turning points for these waves do not exist in the usual sence. It means, that these waves must propagate from a source down and up by decreasing their amplitudes. Hence only the "transition" turning point for the dissipative modes can appear. The details of this question have been discussed by us in a recent paper (Dzhalilov et al. $2000)$ for the $p$-modes leakage problem. The place of the location of the sources of these waves can be taken in those places where the adiabatic approximation fails. Around the upper turning point $z=z_{\mathrm{t}}$ the waves are rather nonadiabatic, where their vertical wavelengh is large.

Thus we have a radiation boundary condition for the dissipative modes: from the point $z=z_{\mathrm{t}}$ (turning point of adiabatic waves) the dissipative waves are radiated. In all directions the amplitudes of these modes must decrease, 
and while setting $\varepsilon \equiv 0$ these modes must disappear. The following solution obeys these conditions:

$$
\begin{aligned}
& \Theta=\Theta_{\mathrm{D}} \approx \frac{T_{0}^{1 / 4}}{\left(1+\varepsilon \varphi_{2}\right)^{5 / 4}} \mathrm{e}^{J-J_{0}}, \\
& J_{0}(z)=\frac{1}{2} \int_{z}^{z_{\mathrm{t}}} \varepsilon G(z) \mathrm{d} z, \quad G(z)=\frac{\varphi_{1}-\psi_{1} \varphi_{2}}{1+\varepsilon \varphi_{2}}, \\
& J(z)=i \int_{z_{\mathrm{t}}}^{z}\left(\frac{1}{\varepsilon}+\varphi_{2}\right)^{1 / 2} \mathrm{~d} z, \text { if } z \geq z_{\mathrm{t}} \\
& J(z)=i \int_{z}^{z_{\mathrm{t}}}\left(\frac{1}{\varepsilon}+\varphi_{2}\right)^{1 / 2} \mathrm{~d} z, \text { if } z \leq z_{\mathrm{t}} .
\end{aligned}
$$

Here the branch with $\sqrt{2 i}=+(1+i)$ is choosen, $\operatorname{Re}(J)<$ 0 , and $T_{0}(z)$ is normalized to $T_{\mathrm{c}}$.

\subsection{Quasi-adiabatic modes}

Now we will search for "slow" quasi-adiabatic wave solutions in the form

$\Theta=\sum_{n=0}^{\infty} \tilde{\varepsilon}^{n} Y_{n}(z)$

where the functions $Y_{n}(z)$ are supposed to be smooth. Inserting this formal solution into Eq. (45) we receive the reccurent differential relations between the $Y_{n}$ functions:

$\Phi_{2} Y_{0}^{\prime \prime}+\Phi_{1} Y_{0}^{\prime}+\Phi_{0} Y_{0}=0$

$\Phi_{2} Y_{n}^{\prime \prime}+\Phi_{1} Y_{n}^{\prime}+\Phi_{0} Y_{n}=-\left(\frac{\mathrm{d}}{\mathrm{d} z}+\varphi_{3}\right) \frac{d^{3}}{d z^{3}} Y_{n-1}$

where $n=1,2, \ldots$ For our aim we are interested in the main value of the solution (56) only. Therefore for small $\varepsilon$ Eq. (57) describes the main properties of the quasiadiabatic waves. To obtain the standard form of this equation we introduce a new variable

$Y_{0}=\exp \left(-\frac{1}{2} \int^{z} \frac{\Phi_{1}}{\Phi_{2}} \mathrm{~d} z\right) W=T_{0}^{3 / 4} \sqrt{a}|d| \mathrm{e}^{J_{0}} W$,

where $J_{0}(z)$ is defined by Eq. (54). Then we have

$W^{\prime \prime}+\left(\lambda^{2} \wp+\tilde{\varphi}\right) W=0$

where $\lambda^{2}=c_{0}^{2} \Lambda / c^{2}, \tilde{\omega}_{0}=\omega R_{\odot} / c_{0}, c_{0}$ is given for the temperature in the solar center, and

$$
\begin{aligned}
\wp(z)= & \frac{\Lambda-\alpha_{0}}{1+\varepsilon \varphi_{2}} \frac{\tilde{\omega}_{0}^{2}}{\Lambda}\left(\frac{N^{2}}{\omega^{2}}-1+\varepsilon{\tilde{k_{\perp}^{2}}}^{2},\right. \\
\alpha_{0}= & 1+\left(æ_{\rho}+\frac{\mathrm{d}}{\mathrm{d} z}\right) \frac{q}{d}+\left(\tilde{\omega} \frac{q}{d}\right)^{2} \approx-q \frac{d^{\prime}}{d^{2}}, \\
\tilde{\varphi}(z)= & \frac{b_{1}\left(a_{1}+\wp_{\mathrm{T}}+b_{1}^{\prime} / b_{1}\right)+\varepsilon \varphi_{0}}{1+\varepsilon \varphi_{2}}- \\
& -\frac{1}{4}\left(\psi_{1}+\varepsilon G\right)^{2}-\frac{1}{2} \frac{\mathrm{d}}{\mathrm{d} z}\left(\psi_{1}+\varepsilon G\right) .
\end{aligned}
$$

Here $\lambda=$ const is a large spectral parameter, and $\tilde{\varphi}(z)$ is a smooth function. The asymptotic theory for differential equations of second order such as Eq. (60) is well developed for real $\wp$. In our case the function $\wp$ is complexvalued with a complex spectral parameter $\omega$. In such a situation the direct application of the standard asymptotic theory to Eq. (60) is impossible. For the complex-valued $\wp$ the turning points of Eq. (60) are shifted from those of the adiabatic Eq. (40). Such small shifts are very important for the solution of the frequency discrepancy problem of helioseismology (Dzhalilov et al. 2000). For our task here the small shifts of the turning points are unimportant. Thus we take for Eq. (60) the same turning points which we have discussed in the section of adiabatic waves. For the solutions we apply the same method as earlier for the $p$-modes (Dzhalilov et al. 2000). We get the solutions

$W=\left(\frac{\xi}{\wp}\right)^{1 / 4}\left[C_{1} A_{i}(-\xi)+C_{2} B_{i}(-\xi)\right], z \leq z_{\mathrm{t}}$,
$\xi=\left(\frac{3}{2} \eta\right)^{2 / 3}, \eta=\lambda \int_{z}^{z_{\mathrm{t}}} \sqrt{\wp} \mathrm{d} z, \xi^{\prime}(z)=-\lambda \sqrt{\frac{\wp}{\xi}}$,

where $\xi^{\prime}\left(z_{\mathrm{t}}\right) \neq 0$ and

$$
\begin{aligned}
W & =\left(-\frac{\xi}{\wp}\right)^{1 / 4}\left[C_{1} A_{i}(\xi)+C_{2} B_{i}(\xi)\right], z \geq z_{\mathrm{t}}, \\
\xi & =\left(\frac{3}{2} \eta\right)^{2 / 3}, \eta=\lambda \int_{z_{\mathrm{t}}}^{z} \sqrt{-\wp} \mathrm{d} z, \xi^{\prime}(z)=-\lambda \sqrt{-\frac{\wp}{\xi}} .
\end{aligned}
$$

Here the regular functions $A_{i}(\xi)$ and $B_{i}(\xi)$ are the Airy functions of first and second kind and $C_{1,2}=$ const. In the derivation of the solutions $(64+66)$ the branch $-1=$ $\exp (i \pi)$ has been taken.

Now we have to determine from the boundary conditions one of the unknown constants $C_{1,2}$. Here we assume the next simplification. In the real solar situation we should include the tunneling of waves through the opaque convective zone to the transparent solar surface. However, in this work we do not complicate the situation by including this important effect. Here we are interested in the eigenoscillation spectrum of the main interior cavity. So our solutions must be finite in the whole domain of integration: $0 \leq z \leq \infty$. In the limit of $z \rightarrow \infty$ we have the asymptotics

$A_{i}(\xi) \approx \frac{1}{2 \sqrt{\pi}} \xi^{-1 / 4} \mathrm{e}^{-\eta}, \quad B_{i}(\xi) \approx \frac{1}{\sqrt{\pi}} \xi^{-1 / 4} \mathrm{e}^{\eta}$,

where $\eta$ is complex and $\operatorname{Re}(\eta) \rightarrow \infty$. From here we have the condition $C_{2}=0$, and the solutions $(64+66)$ are expressed only by the function $A_{i}(\xi)$. For small $\varepsilon$ we have the cavity solution for the quasi-adiabatic waves from Eqs. (56), (59), and (64):

$\Theta=\Theta_{\mathrm{NA}} \approx T_{0}^{3 / 4} \sqrt{a}|d| \mathrm{e}^{J_{0}}\left(\frac{\xi}{\wp}\right)^{1 / 4} A_{i}(-\xi)$,

where $T_{0}(z)$ is normalized to $T_{\mathrm{c}}$. This solution is limited at the boundaries of the convective zone $(d=0)$. Now we can define the general solution of our general Eq. (37) as a superposition of the solutions for the non-adiabatic (Eq. (68)) and the dissipative (Eq. (53)) cases:

$\Theta=C_{1} \Theta_{\mathrm{NA}}+C_{2} \Theta_{\mathrm{D}}$ 
where the new arbitrary constants $C_{1}$ and $C_{2}$ must be determined from the boundary conditions.

\section{Boundary value problem}

In this section we impose physically reasonable boundary conditions to the general solution of (69) to determine the spectrum of the eigenoscillations of the inner cavity.

\subsection{Solar center}

At the solar center $(z=0)$ where $g \rightarrow 0, x_{\mathrm{p}} \rightarrow 0$, and $æ_{\mathrm{T}, \rho}=O(1)$ we apply a rigid boundary condition: $v_{z}(0)=$ 0 . As the function $\Theta(z)$ is finite at the center Eq. (25) reads as $\mathrm{d}\left(p^{\prime} / p_{0}\right) / \mathrm{d} z=0$. Using Eqs. $(27)+(21)$ with dimensionless parameters we have

$\frac{\mathrm{d}}{\mathrm{d} z} \Theta\left(1+\varepsilon \frac{\Theta^{\prime \prime}}{\Theta}\right)=0$.

It is easy to show that for both solutions the conditions $\Theta_{\mathrm{NA}}^{\prime \prime} / \Theta_{\mathrm{NA}} \approx$ const., $\Theta_{\mathrm{D}}^{\prime \prime} / \Theta_{\mathrm{D}} \approx$ const are obeyed. Then the condition (70) is changed to

$\left.\frac{\mathrm{d} \Theta}{\mathrm{d} z}\right|_{z=0}=0$.

Now we insert the general solution (69) into this condition and receive the ratio between the unknown coefficients:

$\frac{C_{2}}{C_{1}}=-\frac{\Theta_{\mathrm{NA}}^{\prime}(0)}{\Theta_{\mathrm{D}}^{\prime}(0)}$.

To obtain the dispersion relation we need one more boundary condition.

\subsection{Bottom of the convective zone}

Let us consider the location of the upper turning point $\left(z=z_{\mathrm{t}}\right)$ : there the equation $N^{2} \approx \omega^{2}$ is fulfilled, from which we may define the parameter $d, d \approx O\left(R_{\odot} \omega^{2} / g\right) \approx$ $10^{-9} \approx 0$. Here we can apply a free boundary condition, because at the bottom of the convective zone the BruntVäisälä frequency and the rotation rate $\Omega(z, \theta)$ change very sharp. (A more realistic approach would be to include the tunneling of the waves.) At the free surface the Lagrangian pressure must be constant, $\mathrm{d} p / \mathrm{d} t=0$, where $p=p_{0}+p^{\prime}$ is the total pressure. From here we have

$v_{z}=-\left.\frac{i \omega c^{2}}{g} \frac{p^{\prime}}{p_{0}}\right|_{z=z_{\mathrm{t}}}$

From Eqs. $(33)+(27)$ we define the next formulae written in dimensionless parameters

$$
\begin{aligned}
a \kappa \frac{p^{\prime}}{p_{0}}= & \Theta+\frac{q \tilde{\omega}^{2}}{d^{2}}\left(1-\frac{N^{2}}{\omega^{2}}+\varepsilon \frac{d^{2}}{d z^{2}}\right) \Theta \\
& +\frac{1}{d}\left(æ_{\rho}-\frac{5}{2} æ_{\mathrm{T}}-\frac{d^{\prime}}{d}+\frac{\mathrm{d}}{\mathrm{d} z}\right)\left(1+\varepsilon \frac{d^{2}}{d z^{2}}\right) \Theta, \\
\frac{v_{z}}{c}=\frac{i \tilde{\omega}}{\kappa d} & {\left[\left(1+\varepsilon \frac{d^{2}}{d z^{2}}\right) \Theta-\kappa q \frac{p^{\prime}}{p_{0}}\right] . }
\end{aligned}
$$

Having in mind that at $z \approx z_{\mathrm{t}}$ we have $a \approx-q d^{\prime} / d^{2}$ and $d^{\prime}<0$; neglecting small values we get from Eqs. $(73)+$ $(75)$

$$
\left(1+\varepsilon \frac{d^{2}}{d z^{2}}\right) \Theta=\kappa q \frac{p^{\prime}}{p_{0}} .
$$

Now excluding $p^{\prime} / p_{0}$ from here and from Eq. (74) we get the second boundary condition

$$
\left.\frac{\mathrm{d} \Theta}{\mathrm{d} z}\right|_{z=z_{\mathrm{t}}} \approx 0 .
$$

Inserting the general solution (69) into this condition we get

$$
\frac{C_{2}}{C_{1}}=-\frac{\Theta_{\mathrm{NA}}^{\prime}\left(z_{\mathrm{t}}\right)}{\Theta_{\mathrm{D}}^{\prime}\left(z_{\mathrm{t}}\right)} .
$$

\subsection{Dispersion relation}

From Eqs. $(72)+(78)$ we get the general dispersion relation

$\Theta_{\mathrm{NA}}^{\prime}(0)=\Theta_{\mathrm{D}}^{\prime}(0) \frac{\Theta_{\mathrm{NA}}^{\prime}\left(z_{\mathrm{t}}\right)}{\Theta_{\mathrm{D}}^{\prime}\left(z_{\mathrm{t}}\right)}$.

To simplify this equation the asymptotic expansions of the Airy functions can be used. At the center of the Sun the arguments of the Airy functions become large: $|\xi|>>1$. Then the main values of the asymptotic expansions yield (Eqs. $(68)+(53))$

$\begin{aligned} \Theta_{\mathrm{NA}}^{\prime}(0) & \simeq-\frac{i \lambda^{2} \wp_{\mathrm{c}}^{1 / 4}}{\sqrt{\pi}} \cos \left(\eta_{0}+\frac{\pi}{4}\right), \\ \Theta_{\mathrm{D}}^{\prime}(0) & \simeq-\frac{i}{\sqrt{\tilde{\varepsilon}}} \exp \left(\frac{i}{\sqrt{\tilde{\varepsilon}}} \int_{0}^{z_{\mathrm{t}}} \frac{T_{0}}{T_{\mathrm{c}}} \mathrm{d} z\right),\end{aligned}$

where $\eta_{0}=\eta(0)$ (Eq. (65)) and $\wp_{\mathrm{c}}=\wp(0) \approx-\tilde{\omega}_{0}^{2}$ (Eq. (61)). At the turning point $z=z_{\mathrm{t}}$ we have

$$
\begin{aligned}
\Theta_{\mathrm{NA}}^{\prime}\left(z_{\mathrm{t}}\right) & \simeq\left(\frac{T_{\mathrm{t}}}{T_{\mathrm{c}}}\right)^{3 / 4}\left(-d^{\prime} q\right)^{1 / 2}\left(\wp^{\prime}\left(z_{\mathrm{t}}\right)\right)^{1 / 6} \lambda^{5 / 6} A_{i}^{\prime}(0), \\
\Theta_{\mathrm{D}}^{\prime}\left(z_{\mathrm{t}}\right) & \simeq-\left(\frac{T_{\mathrm{t}}}{T_{\mathrm{c}}}\right)^{3 / 4} \frac{i}{\sqrt{\tilde{\varepsilon}}}
\end{aligned}
$$

where $T_{\mathrm{t}}=T_{0}\left(z_{\mathrm{t}}\right)$. Now setting Eqs. (80)-(83) into (79) we get

$$
\begin{aligned}
\cos \left(\eta_{0}+\frac{\pi}{4}\right) \simeq & i \sqrt{\pi} A_{i}^{\prime}(0) \frac{\wp^{\prime}\left(z_{\mathrm{t}}\right)\left(-d^{\prime} q\right)^{1 / 2}}{\lambda^{7 / 6} \wp_{\mathrm{c}}^{1 / 4}} \\
& \times \exp \left[\frac{i-1}{\sqrt{2|\tilde{\varepsilon}|}} \int_{0}^{z_{\mathrm{t}}}\left(\frac{T_{0}}{T_{\mathrm{c}}}\right)^{1 / 2} \mathrm{~d} z\right] .
\end{aligned}
$$

The right-hand side of this equation is negligible small as $A i^{\prime}(0)=-0.259, \lambda \gg 1$ (Eq. (60)), $q \approx(\gamma-1) / \gamma$ (Eq. $\left.\left(37^{\prime}\right)\right), d^{\prime}\left(z_{\mathrm{t}}\right) \approx 16$, and mainly $\tilde{\varepsilon} \approx 10^{-8}$ (Eq. (38)). Thus we have finally using Eq. (65)

$\lambda \int_{0}^{z_{\mathrm{t}}} \sqrt{\wp(z)} \mathrm{d} z=\pi\left(n-\frac{3}{4}\right)$. 
We may rewrite this dispersion relation for the $R$-modes in a visually more convenient form

$$
\begin{aligned}
\frac{\omega}{2 \Omega_{\odot}} & =\frac{\beta \bar{k}_{x}}{\bar{k}_{\perp}^{2}+\sigma_{n}^{2}} \\
I\left(\omega, k_{x}\right) & =\int_{0}^{z_{\mathrm{t}}} \frac{N}{2 \Omega_{\odot}} \sqrt{A\left[1-\frac{\omega^{2}}{N^{2}}\left(1-\varepsilon \tilde{k}_{\perp}^{2}\right)\right]} \mathrm{d} z \\
A(z, \omega) & =\frac{1-\alpha_{0} / \Lambda}{1+\varepsilon \varphi_{2}}, \sigma_{n}=\frac{\pi(n-3 / 4)}{I}
\end{aligned}
$$

Here $n=1,2, \ldots$ are the radial node numbers, for the other parameters see Eqs. $\left(37^{\prime}\right)+(62)$. In the adiabatic limit $(\varepsilon=0$ and then $\operatorname{Im}(\omega)=0)$ for very small frequencies $\left(\omega^{2} \ll N^{2}\right)$ and for incompressible motions $\left(c^{2} \rightarrow \infty\right)$ we get the classical dispersion relation for Rossby waves: $\omega \sim \beta k_{x} /\left(k_{\perp}^{2}+k_{z}^{2}\right)$ with $k_{z}^{2}=\sigma_{n}^{2}$.

\section{Instability of 22-year, 4000 -yr, and quasi-biennial oscillations}

Now we can determine the complex eigenfrequencies $\omega=\omega_{n}\left(\beta, k_{x}, k_{y}, \alpha\right)$ from the integral dispersion relation Eq. (86) for the given $\Omega_{\odot}=2.86 \times 10^{-6} \mathrm{~s}^{-1}$. Recall that $\beta$ is the latitudinal gradient of the rotation rate, $\alpha$ is the temperature exponent of the nuclear reaction power, $\Omega \approx \tilde{\Omega}\left(1-\beta y / R_{\odot}\right)$, and $\alpha$ determines the power exponent of the temperature of the nuclear reactions, $Q \sim \rho^{2} T^{\alpha}$ (Eq. (5)). For the $\mathrm{p}-\mathrm{p}$ reactions we will use $\alpha=4$. The dependence of this equation on the free parameter $k_{y}$ is a drawback of this equation. In real situations the waves cannot be progressive across the shear in $y$-direction. In the general case the complex $k_{y}=k_{y}\left(k_{x}, \beta, \omega\right)$ is a solution of the two-dimensional eigenvalue problem. To simplify our task for a very small latitudinal gradient of rotation $\beta$ we have introduced the free parameter $k_{y}$. To simulate the decay of the wave amplitudes towards the pole, in $y$ direction, we shall consider only the case with real $k_{y}^{2}<0$. A negative spectral parameter $k_{y}^{2}$ could also be confirmed by the tidal equation of Laplace (e.g. Lee \& Saio 1997) for very low frequencies. This equation includes the influence of sphericity on the angular dependence of the eigenfunctions, if simple rigid rotation is considered in the frame of the traditional geophysical approximation (which fails near the equator).

Ando (1985) has derived a local dispersion relation for waves around the stellar equator. Solving his Eq. (15) with respect to $k$ (that is our $\left.k_{y}\right)$ for low frequencies $(\omega / 2 \Omega \ll$ 1) we get two solutions: $k^{2}=-m^{2}$ and $k^{2}=-m^{2} N^{2} / 4 \Omega^{2}$, where $m$ is the azimuthal wave number. As $N^{2} / 4 \Omega^{2} \gg 1$, the second solution is strongly damped in $y$-direction. Our case corresponds to the first solution, $k^{2}=-m^{2}$.

From geophysical applications (Pedlosky 1982; Gill 1982) we infer that $k_{y}^{2}\left(k_{x}\right) \sim-k_{x}^{2}$ is obeyed for the ageostrophic wave propagation across the shear flow. Really, it is seen from Fig. 2, that the mode separation with respect to $n$ is essential for the solar situation if the condition $k_{y}^{2} \approx-k_{x}^{2}$ is fulfilled. In Fig. 2 the $\operatorname{Re}\left(\omega\left(k_{y}\right)\right)$

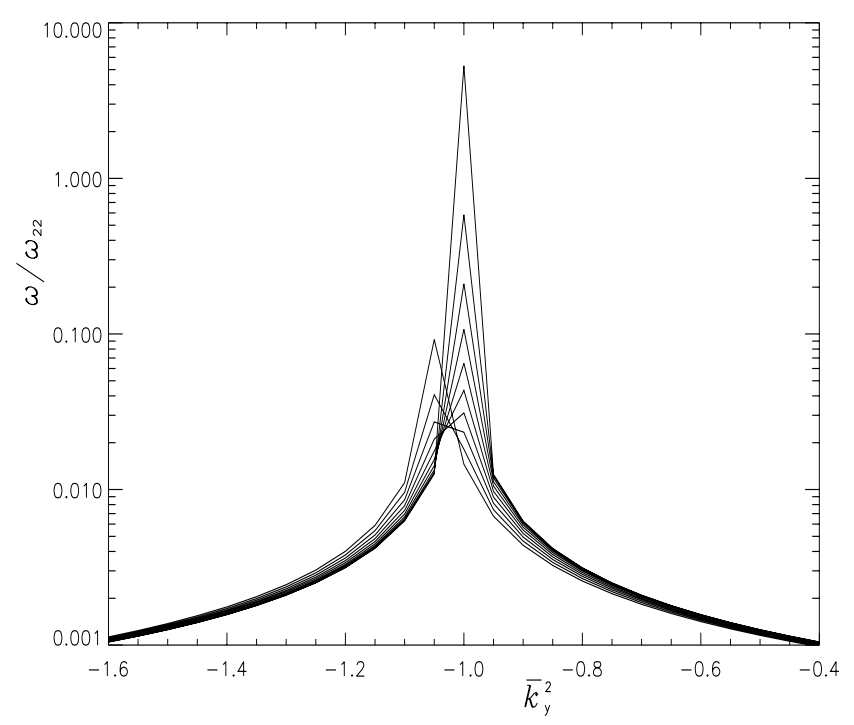

Fig. 2. Mode separation region versus $\bar{k}_{y}=k_{y} R_{\odot}$. On the vertical axis the absolute values of the frequencies normalized to $\omega_{22}$ are shown. The maxima from top to down in the curves correspond to $n=1,3,5, \ldots, 19$. Calculations are done for the case $\bar{k}_{x}=1, \beta=10^{-6}, \eta=10^{-5}$. This figure does not yet show the eigenfrequencies, but the domain of $k_{y}^{2}$ where the $n$-dependence of the frequencies is obvious. This domain is $k_{y}^{2} \approx-k_{x}^{2}$.

dependence is shown for the case $\bar{k}_{x}=1$ and $\beta=10^{-6}$ for example. Here, a very small imaginary part of the frequency is supposed, $\eta \ll 1$, where

$\omega=\omega_{\mathrm{r}}(1+i \eta), \quad \eta=\operatorname{Im}(\omega) / \operatorname{Re}(\omega)$.

In the case $k_{y}^{2} / k_{x}^{2} \neq-1$ we have a continuous spectrum which is physically not interesting. So during this work we have excluded the parameter $k_{y}^{2}$ from our dispersion relation (86) using $k_{y}^{2}=-k_{x}^{2}$. As a result the frequencies depend only on the product $\beta k_{x}$; the dependence on $\alpha$ is very weak.

It follows from Eq. (86) that for a given $\beta k_{x}$ the frequencies $\omega_{n}$ decrease with increasing harmonic number $n$ $\left(\omega \sim 1 / n^{2}\right)$ in the adiabatic case $(\varepsilon=0)$. At very high $n \rightarrow \infty$ we have almost steady motions with a frequency $\omega \rightarrow 0$. Howewer, the situation is changed if dissipation is taken into account, $\varepsilon \neq 0$. In this case small-scale motions are quickly damped, very low frequency oscillations with high $n$ could not become trapped and cannot manifest themselves as eigenmodes. Really, as the nonadiabatic parameter $\varepsilon \approx 1 / \omega$, at very low frequencies the solutions of the initial equations must have a dissipative character. An investigation of the initial equations for a steady flow with $\partial / \partial t=0$ is a separate task. Here we restrict ourselves only to our dispersion relation Eq. (86) with increasing $n$. We can rewrite this equation as $1 / n^{2} \approx \omega / I^{2}$. With decreasing $\omega$ the right-hand part $\omega / I^{2}$ decreases, and then it becomes independent from $\omega$. It means, that $n$ is limited: $n \leq n_{\text {max }}$. Hence, it is a results of the dissipative effects, that only limited modes become trapped. The value of $n_{\max }$ depends on $k_{x}$ and $\beta$. In Fig. 3 the $n_{\max }\left(k_{x}, \beta\right)$ dependence is presented. It follows from this figure that for a 


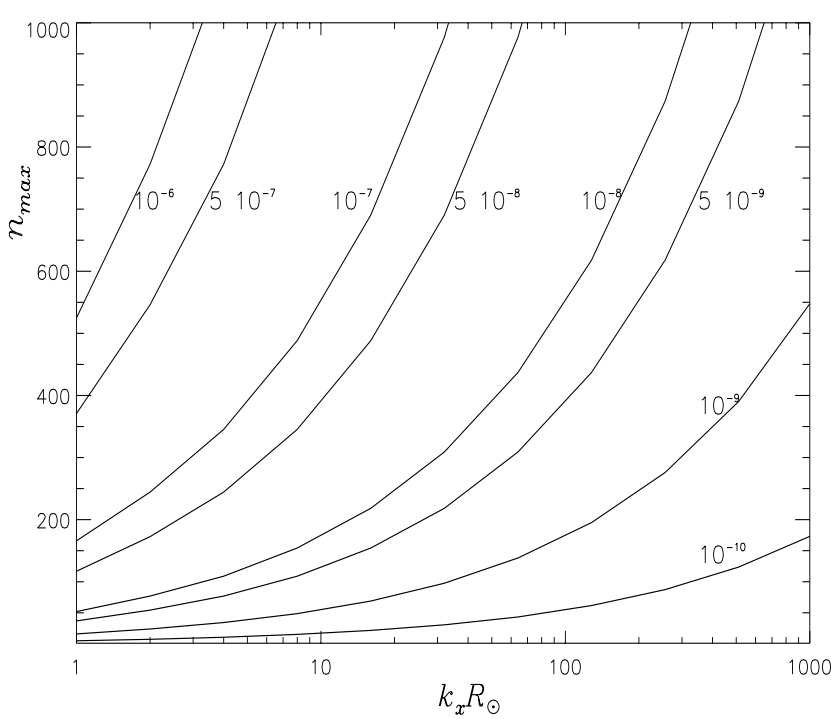

Fig. 3. The harmonic numbers $\left(n_{\max }\right)$ limited by the nonadiabatic effects versus $k_{x}$ and $\beta$. The quantities on the curves correspond to the gradients of the rotation rate $\beta$. Such a limit for high $n$ does not exist for the adiabatic waves.

given $k_{x}$ the number $n_{\max }$ increases strongly by increasing the rotation gradient $\beta$. So, if the solar interior is rotating similar to a solid-body, very long-period oscillations (almost steady flows) should be suppressed. As the values of $n_{\max }$ are sufficiently high, the accuracy of the asymptotic solutions is high.

Now we consider which spectrum of trapped waves with $1 \leq n \leq n_{\max }$ is possible. To calculate the wave spectrum from Eq. (86) we use the standard model of Stix (private communication; Stix \& Slaley 1990) for the solar interior. For the special case $\bar{k}_{x}=k_{x} R_{\odot}=100$ (i.e. $\lambda_{x} \approx 40000 \mathrm{~km}$ ) the $n$-dependence of the real and imaginary parts of the eigenfrequencies are shown in Figs. 4a,b. The calculations were done for different small values of the rotation rate gradient $\beta$, covering a wide range: $\beta=10^{-7}$ $10^{-4}$. This is done because we know from helioseismology only that the $\beta$ parameter is small, but the exact value is not yet known. In Figs. 4a,b $\omega_{\mathrm{r}}=\operatorname{Re}(\omega)$ and $\omega_{i}=\operatorname{Im}(\omega)$ are normalized to the cycle frequency of the 22-year oscillations: $\omega_{22}=0.91 \times 10^{-8} \mathrm{~s}^{-1}=2 \pi(1.45 \mathrm{nHz})$. As expected the frequencies decrease with $n$ and increase with $\beta$ or $k_{x}$. The imaginary parts $\omega_{i}$ oscillate around the zero value: if $\omega_{i}>0$, the waves are unstable and their amplitudes increase with time; in the opposite case, if $\omega_{i} \leq 0$, we have stable/damped waves. In Fig. $4 \mathrm{~b}$ we have two positive maxima: the first corresponds to short-period oscillations of 1-3 yr ("quasi-biennial modes") and the second one to medium-period oscillations of $18-30-y r$ ("22-yr modes"). The position of the quasi-biennial modes versus $n$ is stable and is $\approx 15$. For the 22-yr modes $n_{22}$ is slightly increased with an increase of $\beta$. It is seen from Fig. 4a that $n$ must increase to keep the same frequency with increasing $\beta$. For smaller frequencies this shift is stronger. Figure $4 \mathrm{~b}$ shows that the instability gets stronger if $\beta$ increases: unstable waves become more unstable and damped waves are
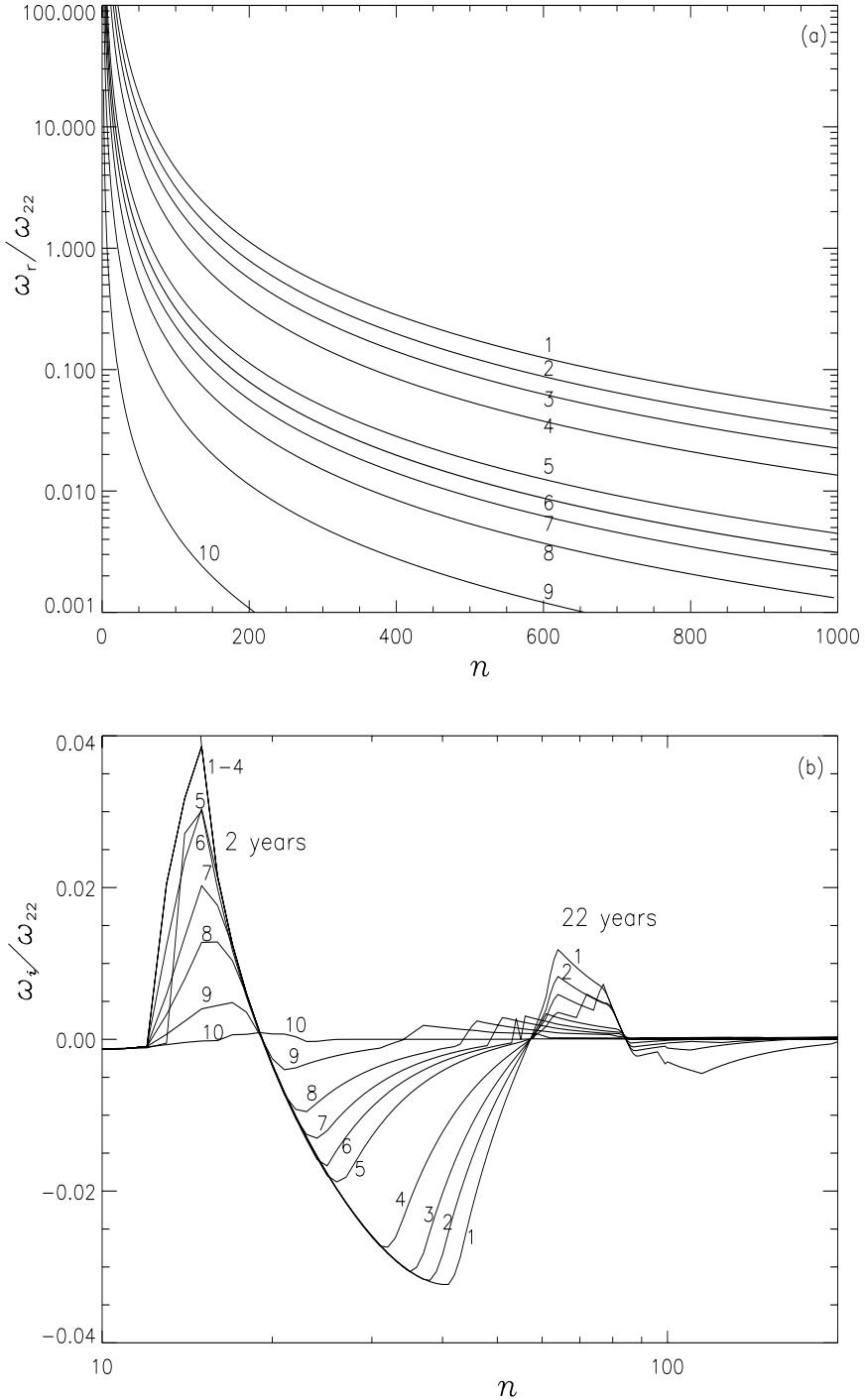

Fig. 4. Real $\left(\omega_{\mathrm{r}}\right)$ and imaginary $\left(\omega_{i}\right)$ parts of the eigenfrequencies (a) and b), respectively) normalized to $\omega_{22}$ versus the radial harmonic number $n$. The numbers on the curves 1 , $2, \ldots, 10$ correspond to different values of the latitudinal gradient of the rotation rate $\beta_{1-10}=10^{-4}, 7 \times 10^{-5}, 5 \times 10^{-5}, 3 \times$ $10^{-5}, 10^{-5}, 7 \times 10^{-6}, 5 \times 10^{-6}, 3 \times 10^{-6}, 10^{-6}, 10^{-7}$. Positive $\omega_{i}$ corresponds to the growth rate of the waves due to the $\varepsilon$ mechanism. For negative $\omega_{i}$ the waves become damped in time due to radiative losses.

stronger damped. Waves with high $n>200$ at very low frequencies also show instability which cannot be shown in Fig. 4b due to the scaling.

Of course, $\beta$ will change with the radius in the real solar radiative interior. Hence, those places, where $\beta$ becomes relatively large, may become sources of unstable waves. Our calculations can easily be generalized for any $k_{x}$ as Eq. (86) is a function of $\beta k_{x}$ only.

To characterize the mode instability, the behavior of the parameter $\eta$ is more important. $\eta$ is the growth rate (increment) of the instability of the modes if $\eta>0$ and the damping rate (decrement) if $\eta<0$. In Fig. 5 we present the $\eta\left(\omega_{\mathrm{r}}\right)$ dependence for the whole range of 


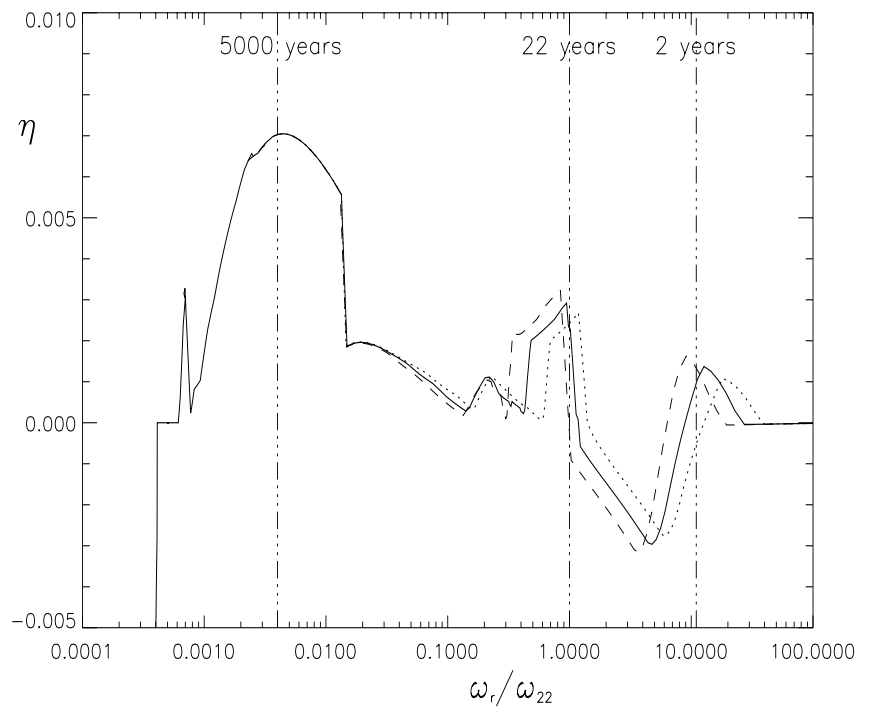

Fig. 5. Growth rate of the instabilities of the eigenmodes of the differentially rotating Sun. Three modes with periods of $\approx 1-3 \mathrm{yr}, 18-30 \mathrm{yr}$ (these ranges depend on $\beta \bar{k}_{x}$ ) with a small additional peak at $100 \mathrm{yr}$, and 1500-20000 yr (independent of $\left.\beta \bar{k}_{x}\right)$ become maximum unstable $(\eta>0)$ for high orders $n$. The solid, dashed, and dotted lines correspond to the small latitudinal gradients of the rotation rate of $\beta=7 \times 10^{-6}, 5 \times$ $10^{-6}$, and $10^{-5}$ at $\bar{k}_{x}=100$.

frequencies $\left(\omega_{\mathrm{r}}<\Omega_{\odot}\right)$ for which our asymptotic theory is valid. We have three distinguished global maxima for the growth rate $\eta>0$ which correspond to period ranges of $\approx 1-3 \mathrm{yr}, 18-30 \mathrm{yr}$ with a small additional peak at $100 \mathrm{yr}$, and 1500-20000 yr ("4000-yr modes") of the eigenmodes. These modes have radial node numbers near $n=850,60$, and 15, respectively. It was already mentioned, that for the unstable modes the value of $n$ is slightly changed with a change of $\beta$. So for the unstable modes $n$ is high and the asymptotic results are reliable. The growth rates of the 22-yr oscillations are always greater than those of the quasi-biennial modes. The characteristic growing time for the 22-year modes is $\approx 1000$ yr as $\eta \sim 0.003$.

Now we consider in which range of $n$ and $\beta \bar{k}_{x}$ the unstable modes are located. For this aim we fix the frequency as $\omega_{\mathrm{r}}=\omega_{22}=0.91 \times 10^{-8} \mathrm{~s}^{-1}$, and for a given $n \leq n_{\max }$ we find the complex root of the dispersion relation Eq. (86). The parameters $\beta \bar{k}_{x}$ and $\eta$ correspond to this root. The same calculations were done for the 2 -yr oscillations. The results are shown in Fig. 6. Here the solid lines correspond to the 22-yr and the dashed lines to the quasi-biennial modes. A sharp increase of $n$ to $n_{\max }$ with $\beta \bar{k}_{x}$ in Fig. 6a indicates some upper limit for the parameter $\beta \bar{k}_{x}$. For the 22-yr and 2-yr modes these limits are approximately $3 \times 10^{-3}$ and $3 \times 10^{-2}$, respectively. Figure $6 \mathrm{~b}$ (where the increment/decrement is presented) shows for which values of $\beta \bar{k}_{x}$ and $n$ the 22-yr and 2-yr modes become unstable. Two maxima for each mode appear in $\eta$. These are for the 22-year modes:

$n=15, \quad \beta \bar{k}_{x}=9 \times 10^{-5}, \quad \eta=0.004$ and

$n=55, \quad \beta \bar{k}_{x}=7 \times 10^{-4}, \quad \eta=0.003$;
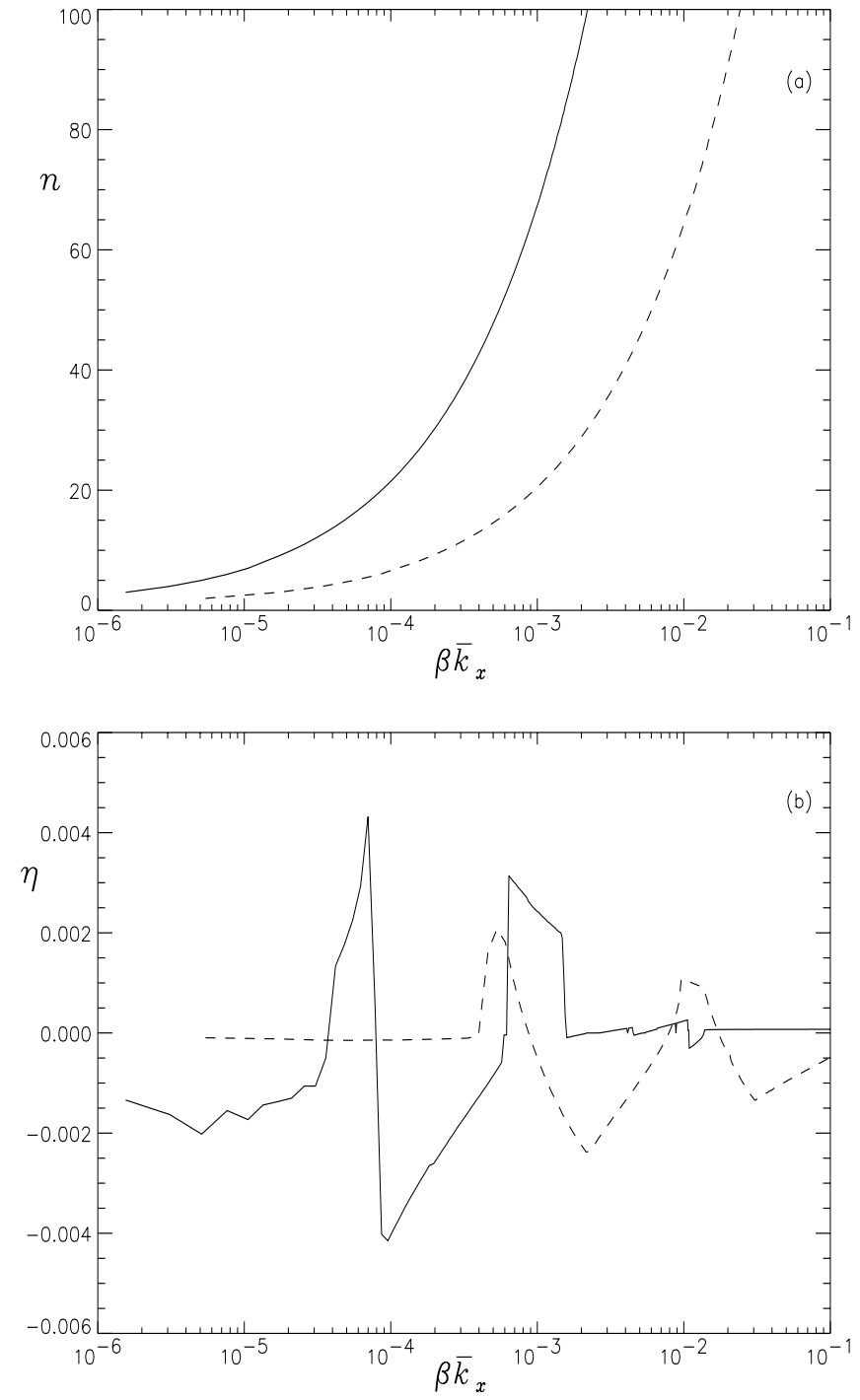

Fig. 6. The existence area of the 22-yr (solid lines) and 2-yr (dashed lines) eigenmodes versus the parameters $n, \beta \bar{k}_{x}$, and $\eta$. Figure $6 \mathrm{a}$ shows that the wavenumber and the rotation gradients $\left(\beta \bar{k}_{x}\right)$ are limited as $n \leq n_{\max }$. The two narrow maxima of the growth rate $\eta$ in Fig. $6 \mathrm{~b}$ indicate that for the strongly fixed values of $\beta \bar{k}_{x}$ the 22-yr and 2-yr modes become unstable (see text).

for the 2-yr modes:

$n=14, \quad \beta \bar{k}_{x}=5 \times 10^{-4}, \quad \eta=0.002$ and

$n=65, \quad \beta \bar{k}_{x}=10^{-2}, \quad \eta=0.001$.

These estimates could be used to define a possible value of $\beta$ inside of the Sun. This will be possible if we can identify these modes from observations. For instance, at $\bar{k}_{x} \approx 100$ (sunspot scale) a gradient of the rotation rate with $\beta=9 \times 10^{-7}$ or $\beta=7 \times 10^{-6}$ is needed to excite the 22-yr modes. Here the possibility of an excitation of both the $n=15$ and the $n=55$ modes is not excluded if $\beta$ is changed with the radius. A better way to define $\beta$ would be to identify both the 22 -yr and the quasi-biennial modes, with different $k_{x}$ at the surface of the Sun. 


\section{Conclusions}

In the present paper we have shown that toroidal eddy flows which are degenerated in a non-rotating fluid can become a reservoir of various branches of oscillatory modes when the degeneracy is removed by rotation. The mechanism depends on the condition for the existence and alteration of the relative vorticity as well as on the stellar rotation rate and its gradient. Apparently at least for slowly rotating stars $\left(\Omega<\Omega_{\mathrm{g}}\right)$ the rotation waves could be divided into two types: $r$-modes with high frequencies $(\omega \leq \Omega)$ which are independent from the inner structure and mainly caused by geometrical effects, and the $R$-modes with low frequencies $(\omega \ll \Omega)$ which depend on the inner structure and are considered in the present paper. This classification is similar to that of $f$ - and $g$ modes or to that of surface and body tube modes of magnetic cylinders. Note that the properties of Coriolis forces and ponderomotive forces in MHD are very similar to each other. Both rotation modes are prototypes of the geophysical Rossby waves.

We investigated the instability problem of the $R$-modes sustained by a very small latitudinal gradient $\beta$ of the rotation rate in the solar radiative interior. The problem has been solved for a realistic solar model without an arbitrary choice of free parameters except $\beta k_{x}$, the product of $\beta$ and the horizontal wave number. Among the eigenoscillations three modes with periods of $\approx 1-3 \mathrm{yr}, 18-30 \mathrm{yr}$, and 1500-20000yr turn out to be maximum unstable to the $\varepsilon$-mechanism. Here the smoothing effect is the radiative damping. All of these instabilities are in the range of high radial node numbers $n$ which indicates that the applicability of the asymptotic solution is satisfied.

The 22-yr modes with a growing time of $\approx 1000$ yr are of particular interest with respect to the solar activity cycle problem. In the simpler case when adiabatic $R$-modes are considered in an incompressible fluid, $\sigma_{n}$ in Eq. (86) is independent of the wave number and of the frequency for very low frequencies. Then in the azimuthal direction the phase and group velocities are

$v_{p x} \approx \frac{\beta}{\bar{k}_{\perp}^{2}+\sigma_{n}^{2}}, \quad v_{g x} \approx-\beta \frac{\bar{k}_{x}^{2}-\bar{k}_{y}^{2}-\sigma_{n}^{2}}{\left(\bar{k}_{\perp}^{2}+\sigma_{n}^{2}\right)^{2}}$,

respectively, where the velocities are normalized to $\Omega_{\odot} R_{\odot}$ $\approx 1.6 \mathrm{~km} \mathrm{~s}^{-1}$. In our case $\beta>0$, i.e. the angular velocity is decreasing towards the pole, similar to the behavior at the solar surface. The $x$-axis in our coordinate system is directed opposite to the direction of rotation, $v_{p x}>0$ and $v_{g x}<0$, moreover, $k_{y}^{2} \approx-k_{x}^{2}$ and for finite $n$ we have $\bar{k}_{x}^{2}>$ $\sigma_{n}^{2}$ (see Fig. 2). Then the retrograde $R$-modes transport energy along the direction of rotation. Our treatment in a Cartesian coordinate system does not allow to determine the direction of energy transport by wave packets relative to the equator in the meridian plane. The estimate of $k_{y}^{2} \approx$ $-k_{x}^{2}$ is crude, and to determine the exact dependence on $k_{y}=k_{y}\left(k_{x}, \omega, \beta\right)$ the $2 \mathrm{D}$ boundary value problem must be solved.
A nice property of the Rossby waves is that every monochromatic mode is a solution of the full nonlinear hydrodynamic equations. It means, that we should expect the development of nonlinear $R$-modes with large amplitudes. We could also expect that just in this nonlinear regime the toroidal magnetic flux will be lifted from the upper boundary of the cavity (the tachocline) to the surface. The energy release of the nonlinear waves could be accomplished by magnetic reconnection. Here it is possible that toroidal currents are generated via a twist of toroidal magnetic field lines by the cyclonic flows of regular $R$-modes with fixed characteristics. Parker (1955) as well as Steenbeck et al. (1966; see also Krause \& Rädler 1980) have suggested for the dynamo process that such a mechanism, the $\alpha$-effect, is working by turbulent motions under the influence of Coriolis forces.

Our present model points out the possibility of forced oscillations instead of a self-excited dynamo to solve the solar cycle problem, and this with the correct period of 22 yr. Similar ideas are due to Tikhomolov (2001) who has recently suggested a hydrodynamic driving of the 11-yr sunspot cycle. There is still a smaller peak of the growth rate (Fig. 5) at 100 years; such a period is observed as a modulation of the $11 / 22$ yr cycles.

We expect that in a non-linear stage of our model a huge toroidal magnetic field of $\approx 10^{5} \mathrm{G}$ will no longer be required to cause a buoyant rise of magnetic flux tubes, because the external nonzero upflow produced by the regular vortical $R$-modes could trigger the eruption of stable magnetic flux tubes stored in the overshoot region. However, we did not yet calculate the details of the rise of the flux tubes, thus the problem of their appearance at the surface with small tilt angles and at low latitudes remains still unresolved.

There is observational evidence for the short-period oscillations as well: from helioseismic sounding Howe et al. $(2000,2001)$ have recently discovered variations of solar rotation with a period of $1.3 \mathrm{yr}$ in the lower convective zone. Quasi-two year modes are very likely seen regularly in various solar data (e.g. Waldmeier 1973; Akioka et al. 1987; Rivin \& Obridko 1992). The existence of two magnetic cycles (the main 22-yr and the quasi-biennial period) on the Sun has been reported by Benevolenskaya $(1996,1998)$. So far the origin of these modes was not yet clear. Terrestrial quasi-biennial oscillations have been clearly seen in tropical meteorological radiosonde data, and a possible solar origin by related phenomena in the solar interior, Rossby waves in particular, has been discussed as well (McIntire 1994).

The long-period oscillations in the broad range $1.5 \times$ $10^{3}-2.0 \times 10^{4} \mathrm{yr}$, with a maximum growth rate around $4500 \mathrm{yr}$, could be the cause of abrupt changes of the global terrestrial climate in the past: Dansgaard-Oeschger events, these are abrupt onsets of warm periods during the last ice age, had mean distances of $4500 \mathrm{yr}$, but they were distributed over a larger period range, similar to that in our model, with shortest distances often around 1500 years (see, e.g., Ganopolski \& Rahmstorf 2001). These events 
were caused by changes of the thermohaline circulation of the ocean, which in its turn were probably triggered by changes in the solar energy output.

Acknowledgements. Michael Stix kindly provided detailed tables from his internal solar model calculations. The critical comments and suggestions by Kris Murawski, Karl-Heinz Rädler, and the referee J. Andrew Markiel helped to improve earlier versions of the paper. The authors gratefully acknowledge financial support of the present work by the German Science Foundation (DFG) under grant No. 436 RUS 113/560/1-1 and -3, by the German Federal Ministry of Education and Research through the German Space Research Center (DLR) under grant No. 50QL 96019, and by the Russian Foundation for Basic Research (RFBR) under grant No. 00-02-16271.

\section{References}

Akioka, M., Kubota, J., Ichimoto, K., et al. 1987, Solar Phys., 112,313

Ando, H. 1985, PAST, 37, 47

Baker, N., \& Kippenhahn, R. 1959, Z. Astrophys., 48, 140

Benevolenskaya, E. E. 1996, Solar Phys., 167, 47

Benevolenskaya, E. E. 1998, ApJ, 509, L49

Brandenburg, A. 1994, in Lectures on solar and planetary dynamos, ed. M. R. E. Proctor, \& A. D. Gilbert (Cambridge Univ. Press), 117

Brayn, G. H. 1889, Phil. Trans. R. Soc. London A, 180, 187

Caligari, P., Schüssler, M., \& Moreno-Insertis, F. 1998, ApJ, 502,481

Cattaneo, F., \& Hughes, D. W. 1996, Phys. Rev. E., 54, R4532

Chaplin, W. J., Christensen-Dalsgaard, J., Elsworth, Y., et al. 1999, MNRAS, 308, 405

Charbonneau, P., \& MacGregor, K. B. 1993, ApJ, 417, 762

Charbonneau, P., \& MacGregor, K. B. 1997, ApJ, 486, 502

Cowling, T. G. 1953, in The Sun, ed. G. P. Kuiper (Univ. Chicago), 565

Dzhalilov, N. S., Staude, J., \& Arlt, K. 2000, A\&A, 361, 1127

Fan, Y., Fisher, G. H., \& Deluca, E. E. 1993, ApJ, 405, 390

Fritts, D. C., Vadas, S. L., \& Andreassen, O. 1998, A\&A, 333, 343

Ganopolski, A., \& Rahmstorf, S. 2001, Nature, 409, 153

Gill, A. E. 1982, Atmosphere-Ocean Dynamics (Acad. Press, Univ. of Cambridge, UK)

Gilman, P. A. 1969, Solar Phys., 8, 316

Gilman, P. A. 1992, in The Solar Cycle, ed. K. L. Harvey, ASP Conf. Ser., 27, 241

Gough, D. O. 1980 in Nonradial and Nonlinear Stellar Pulsation, ed. H. A. Hill, \& W. A. Dziembowski (SpringerVerlag, Berlin), 273

Gough, D. O. 1985, in Future Missions in Solar, Heliospheric and Space Plasma Physics, ESA SP-235, ed. E. Rolfe, \& B. Battrick (ESA Publ. Division, Noordwijk), 183

Gough, D. 1997, Nature, 388, 324

Howe, R., Christensen-Dalsgaard, J., Hill, F., et al. 2000, Science, 287, 2356

Howe, R., Christensen-Dalsgaard, J., Hill, F., et al. 2001, in Proc. SOHO $10 /$ GONG 2000 Workshop Helio- and Asteroseismology at the Dawn of the Millenium, ESA SP164,45
Kosovichev, A. G. 1996, ApJ, 469, L61

Krause, F., \& Rädler, K.-H. 1980, Mean Field Magnetohydrodynamics and Dynamo Theory (Pergamon, Oxford)

Kumar, P., \& Quataert, E. J. 1997, ApJ, 475, L143

Kumar, P., Talon, S., \& Zahn, J. P. 1999, ApJ, 520, 859

Lee, U., \& Saio, H. 1997, ApJ, 491, 839

Levy, E. H. 1992, in Cool Stars, stellar systems, and the Sun, ed. M. S. Giampapa, \& J. A. Bookbinder, ASP Conf. Ser., 26,223

Lockitch, K. H., \& Friedman, J. L. 1999, ApJ, 521, 764

Markiel, J., \& Thomas, J. H. 1999, ApJ, 523, 827

McIntire, M. 1994, in The Solar Engine and Its Influence on Terrestrial Atmosphere and Climate, NATO ASI Ser. I 25, ed. E. Nesme-Ribes (Springer-Verlag, Berlin-Heidelberg), 293

Mestel, L., \& Weiss, N. O. 1987, MNRAS, 226, 123

Moffatt, H. K. 1978, Magnetic Field Generation in Electrically Conducting Fluids (Cambridge Univ., Cambridge)

Oraevsky, V. N., \& Dzhalilov, N. S. 1997, Astron. Rep., 41, 91

Papaloizou, J., \& Pringle, J. 1978, MNRAS, 182, 423

Parker, E. N. 1955, ApJ, 122, 293

Parker, E. N. 1993, ApJ, 408, 707

Paterno L., Sofia, S., \& DiMauro, M. P. 1996, A\&A, 314, 940

Pedlosky, J. 1982, Geophysical Fluid Dynamics (SpringerVerlag, New York- Heidelberg-Berlin)

Provost, J., Berthomieu, G., \& Rocca, A. 1981, A\&A, 94, 126

Ringot, O. 1998, A\&A, 335, L89

Rivin, Y. R., \& Obridko, V. N. 1992, Astron. Zh., 69, 1083

Rüdiger, G., \& Arlt, R. 2000, in Advances in nonlinear dynamos, The Fluid Mechanics of Astrophysics and Geophysics, ed. A. Ferriz-Mas, \& M. M. Jimenez, 8, Chapter 6, in press

Saio, H. 1982, ApJ, 256, 717

Schatzman, E. 1993, A\&A, 279, 431

Schmitt, D. 1993, in The Cosmic Dynamo, ed. F. Krause, K.-H. Rädler, \& G. Rüdiger (Kluwer, Dordrecht), 1

Shore, S. N. 1992, An Introduction to Astrophysical Hydrodynamics (Academic Press, Inc. San Diego, Calif., USA)

Smeyers, P., Craeynest, D., \& Martens, L. 1981, Astrophys. Space Sci., 78, 483

Spiegel, E. A., \& Zahn, J. P. 1992, A\&A, 265, 106

Steenbeck, M., Krause, F., \& Rädler, K.-H. 1966, Z. Naturforsch., 21a, 369

Stix, M. 1976, A\&A, 47, 243

Stix, M. 1991, Geophys. Astrophys. Fluid Dyn., 62, 211

Stix, M., \& Skaley, D. 1990, A\&A, 232, 234

Tikhomolov, E. 2001, Solar Phys., 199, 165

Tikhomolov, E. M., \& Mordvinov, V. I. 1996, ApJ, 472, 389

Tomczyk, S., Schou, J., \& Thompson, M. J. 1995, ApJ, 448, L57

Unno, W., Osaki, Y., Ando, H., Saio, H., \& Shibahashi, H. 1989, Nonradial Oscillations of Stars (Univ. Tokio Press, Tokyo)

Waldmeier, M. 1973, Solar Phys., 28, 389

Weiss, N. O. 1994, in Lectures on solar and planetary dynamos, ed. M. R. E. Proctor, \& A. D. Gilbert (Cambridge Univ. Press), 59

Wolff, C. L., \& Bizard, J. B. 1986, Solar Phys., 105, 1

Wolff, C. L. 1997, ApJ, 486, 1058

Wolff, C. L. 1998, ApJ, 502, 961

Wolff, C. L. 2000, ApJ, 531, 591

Zahn, J. P., Talon, S., \& Matias, J. 1997, A\&A, 322, 320 